\title{
SCHEMES OF A FINITE PROJECTIVE PLANE AND THEIR EXTENSIONS
}

\author{
S. EVDOKIMOV AND I. PONOMARENKO
}

\begin{abstract}
There are several schemes (coherent configurations) associated with a finite projective plane $\mathcal{P}$. In the paper, a new scheme is constructed, which, in a sense, contains all of them. It turns out that this scheme coincides with the 2extension of the nonhomogeneous scheme of $\mathcal{P}$ and is uniquely determined up to similarity by the order $q$ of $\mathcal{P}$. Moreover, for $q \geq 3$, the rank of the scheme does not depend on $q$ and equals 416 . The results obtained have interesting applications in the theory of multidimensional extensions of schemes and similarities.
\end{abstract}

\section{$\S 1$. INTRODUCTION}

A projective plane is a triple consisting of a set of points, a set of lines, and an incidence relation between their elements, the defining property of which is the following: any two distinct points (respectively, lines) are incident to a unique line (respectively, point). To avoid degenerate cases, usually a certain nondegeneracy condition is also imposed. An isomorphism of projective planes is a bijection taking the points (respectively, lines) of one plane to the points (respectively, lines) of the other and preserving the incidence relation.

In this paper we deal with finite projective planes. For such a plane, there exists a positive integer $q$, the order of the plane, such that each point (respectively, line) is incident to exactly $q+1$ lines (respectively, points), and the number of points equals the number of lines and equals $q^{2}+q+1$ (see [14]). For any prime power $q>1$, the projective plane whose points and lines are those of a 3-dimensional linear space over a Galois field of order $q$ with the incidence relation defined by inclusion, is called a Galois plane of order $q 11$ The Galois planes are precisely the finite projective planes for which the Desargues theorem is true. Though there are a lot of non-Desarguesian finite projective planes, up to now it is not known if there exists such a plane of prime order.

There are several schemes (coherent configurations) associated with a finite projective plane $\mathcal{P}$ : a scheme of type $\left[\begin{array}{cc}2 & 2 \\ 2\end{array}\right]$ (nonhomogeneous scheme), a scheme of a distanceregular graph of diameter 3 (homogeneous scheme), and schemes of flags and antiflags 13, 4, 12, 8. In this paper we construct a new scheme that, in a sense, contains all of them. The underlying set of it equals $V^{2}$, where $V$ is the set of elements (the points and lines) of the plane $\mathcal{P}$; in the Desarguesian case it is a fusion of the scheme corresponding to the coordinatewise action of the group $\operatorname{Aut}(\mathcal{P})$ on $V^{2}$. Moreover, it turns out that in the sense of [6] our scheme is none other than the 2-extension of the nonhomogeneous

2000 Mathematics Subject Classification. Primary 05C25, 51A05.

Key words and phrases. Projective plane, Galois plane, scheme, graph.

The first author was partially supported by RFBR (grants 07-01-00485 and 06-01-00471).

The second author was partially supported by RFBR (grants 07-01-00485 and 05-01-00899) and by the grant NS-4329.2006.1.

${ }^{1}$ Here the "plane of order 1" (triangle) is also treated as a Galois plane. 
scheme of $\mathcal{P}$. This fact has interesting applications in the theory of multidimensional extensions of schemes and similarities.

The basis relations of the new scheme mentioned above will be constructed with the help of a special complete colored graph $\Gamma$ with a vertex set $\Omega$ such that

$$
\Xi \subset \Omega \subset \Xi \cup \Xi^{(2)},
$$

where $\Xi$ is the set consisting of four distinct commuting variables $\xi_{1}, \xi_{2}, \xi_{3}, \xi_{4}$, and $\Xi^{(2)}$ is the symmetric square of $\Xi$, the elements of which are written as products of two distinct variables. The colors are called types and come from the types of elements and relations among them in a projective plane. Each vertex of $\Gamma$ has type $\mathfrak{p}$ (point) or $\mathfrak{l}$ (line), whereas each edge has type $\mathfrak{e}$ (equality), $\mathfrak{e}^{\prime}$ (inequality), $\mathfrak{i}$ (incidence) or $\mathfrak{i}^{\prime}$ (nonincidence). We also assume some natural compatibility conditions: (a) the edge between vertices of coinciding (respectively, distinct) types must have type $\mathfrak{e}$ or $\mathfrak{e}^{\prime}$ (respectively, $\mathfrak{i}$ or $\mathfrak{i}^{\prime}$ ), (b) if an edge between variables $\xi_{i}$ and $\xi_{j}$ is of type $\mathfrak{e}^{\prime}$, then $\xi_{i} \xi_{j} \in \Omega$ and the edge between $\xi_{i}$ and $\xi_{i} \xi_{j}$ is of type i. Any such graph $\Gamma$ is called a $\Xi$-configuration.

Let $x=\left(x_{1}, \ldots, x_{4}\right)$, where $x_{i} \in V$ for all $i$. With $x$ we associate a $\Xi$-configuration as follows. Let $\Omega$ be the union of $\Xi$ and the set of all $\xi_{i} \xi_{j} \in \Xi^{(2)}$ such that $x_{i}$ and $x_{j}$ are distinct points or distinct lines in $\mathcal{P}$. The type of a vertex $\xi \in \Omega$ (respectively, an edge between $\xi$ and $\eta$ ) is defined to be the type of the element $f(\xi)$ (respectively, the relation between $f(\xi)$ and $f(\eta))$ in the plane $\mathcal{P}$, where $f(\xi)=x_{i}$ for $\xi=\xi_{i}$ and $f(\xi)$ equals the (unique) element of $\mathcal{P}$ incident to both $x_{i}$ and $x_{j}$ for $\xi=\xi_{i} \xi_{j}$. The graph $\Gamma$ constructed in this way is denoted by $\operatorname{Der}(x)=\operatorname{Der}(x, \mathcal{P})$. For a $\Xi$-configuration $\Gamma$, set

$$
R(\Gamma, \mathcal{P})=\left\{x \in V^{4}: \operatorname{Der}(x)=\Gamma\right\} .
$$

Clearly, when $\Gamma$ runs over all $\Xi$-configurations, the nonempty sets of this kind form a partition $\Pi$ of $V^{4}$ called the derivative partition associated with $\mathcal{P}$. Obviously, for a given set $R \in \Pi$, the graph $\operatorname{Der}(x)$ does not depend on the choice of $x \in R$. We denote this graph by $\operatorname{Der}(R)$ and set

$$
\operatorname{Der}(\mathcal{P})=\{\operatorname{Der}(R): R \in \Pi\} .
$$

Below, classes of the partition $\mathcal{P}$ are identified with binary relations on $V^{2}$ via the natural bijection from $V^{4}$ to $V^{2} \times V^{2}$ that takes $\left(x_{1}, x_{2}, x_{3}, x_{4}\right)$ to $\left(\left(x_{1}, x_{2}\right),\left(x_{3}, x_{4}\right)\right)$.

Theorem 1.1. Let $\mathcal{P}$ be a finite projective plane of order $q$ and $\Pi$ the derivative partition associated with $\mathcal{P}$. Then:

1) the pair $\left(V^{2}, \Pi\right)$ is a scheme;

2) the set $\operatorname{Der}(\mathcal{P})$ does not depend on $\mathcal{P}$ for a fixed $q$, and even does not depend on $q$ for sufficiently large $q$;

3) for given $R, S, T \in \Pi$, the intersection number $c_{R, S}^{T}$ of the scheme $\left(V^{2}, \Pi\right)$ equals $f(q)$, where $f(x)$ is a polynomial with integral coefficients depending only on $\operatorname{Der}(R), \operatorname{Der}(S)$, and $\operatorname{Der}(T)$ (but not depending on $\mathcal{P}$ ).

The $\Xi$-configurations belonging to the set $\operatorname{Der}(\mathcal{P})$ are described in the table of the Appendix. It follows that this set does not depend on $q$ for $q \geq 3$, and the cardinality of $\Pi$ equals 200, 400, and 416 for $q=1, q=2$, and $q \geq 3$, respectively. The reflexive relations of $\Pi$ are given by the eight $\Xi$-configurations uniquely determined by their restrictions to the set $\left\{\xi_{1}, \xi_{2}\right\}$ :

$$
\begin{array}{lccc}
\mathfrak{p} \frac{\mathfrak{e}}{\mathfrak{p}} & \mathfrak{p} \frac{\mathfrak{e}^{\prime}}{\mathfrak{p}} & \mathfrak{p} \frac{\mathfrak{i}}{\mathfrak{l}} & \mathfrak{p} \frac{\mathfrak{i}^{\prime}}{\mathfrak{l}} \\
\mathfrak{l} \stackrel{\mathfrak{e}}{ } \mathfrak{l} & \mathfrak{l} \frac{\mathfrak{e}^{\prime}}{-} \mathfrak{l} & \mathfrak{l} \stackrel{\mathfrak{i}}{-} \mathfrak{p} & \mathfrak{l} \frac{\mathfrak{i}^{\prime}}{} \mathfrak{p}
\end{array}
$$


Thus, the scheme $\left(V^{2}, \Pi\right)$ has eight homogeneity sets:

$$
\begin{aligned}
E_{\mathfrak{t}} & =\left\{(x, y) \in V_{\mathfrak{t}} \times V_{\mathfrak{t}}: x \text { is equal to } y\right\}, & & E_{\mathfrak{t}}^{\prime}=\left(V_{\mathfrak{t}} \times V_{\mathfrak{t}}\right) \backslash E_{\mathfrak{t}}, \\
I_{\mathfrak{t}} & =\left\{(x, y) \in V_{\mathfrak{t}} \times V_{\mathfrak{t}^{\prime}}: x \text { is incident to } y\right\}, & & I_{\mathfrak{t}}^{\prime}=\left(V_{\mathfrak{t}} \times V_{\mathfrak{t}^{\prime}}\right) \backslash I_{\mathfrak{t}},
\end{aligned}
$$

where $\mathfrak{t} \in\{\mathfrak{p}, \mathfrak{l}\}, \mathfrak{t}^{\prime}$ is determined by the condition $\left\{\mathfrak{t}, \mathfrak{t}^{\prime}\right\}=\{\mathfrak{p}, \mathfrak{l}\}$, and $V_{\mathfrak{p}}$ and $V_{\mathfrak{l}}$ are the points and the lines of $\mathcal{P}$. These eight sets, viewed as binary relations on $V$, form a partition $\mathcal{R}$ of $V^{2}$, and the pair $(V, \mathcal{R})$ is exactly the scheme introduced by D. Higman in [13. We call $(V, \mathcal{R})$ and $\left(V^{2}, \Pi\right)$ the (nonhomogeneous) scheme and the derivative scheme of the projective plane $\mathcal{P}$, respectively. The mapping $x \mapsto(x, x)$ takes the basis relations of the former scheme to the relations $R(\Gamma, \mathcal{P})$, where $\Gamma$ runs over the $\Xi$ configurations obtained from the above eight $\Xi$-configurations by interchanging $\xi_{2}$ and $\xi_{3}$. Thus, the nonhomogeneous scheme is isomorphic to the restriction of the derivative scheme to $E_{\mathfrak{p}} \cup E_{\mathfrak{l}}$. Similarly, one can see that the restrictions of the derivative scheme to $I_{\mathfrak{p}}$ and $I_{\mathfrak{p}}^{\prime}$ are the scheme of flags (see [12]) and an extension of the scheme of antiflags, respectively (see [8]).

From Theorem 1.1 it follows that for a sufficiently large $q$ equal to the order of a finite projective plane $\mathcal{P}$, there exists a uniquely determined noncommutative algebra over $\mathbb{R}$ of dimension 416, the adjacency algebra of the derivative scheme of $\mathcal{P}$. The elements of the standard linear basis of it are parametrized by the $\Xi$-configurations from the set $\operatorname{Der}(\mathcal{P})$, not depending on $q$ by statement 2 ) of the theorem; the structure constants are given by a tensor $T(q)$, the entries of which are determined by statement 3 ). Since any prime power is the order of some projective plane, for every real $q>0$ there exists an algebra with structure constant tensor $T(q)$. Moreover, this algebra can be regarded as a generalized $C$-algebra in the sense of $[1$.

Theorem 1.1 also has interesting applications in the theory of $m$-extensions of schemes and similarities, as developed in [7, 5, 6, 2]. By definition, the $m$-extension of a scheme $\mathcal{C}$ on $V$ is a special scheme on the set $V^{m}$, whereas the $m$-extension of a similarity between schemes $\mathcal{C}$ and $\mathcal{C}^{\prime}$ is a special similarity between their $m$-extensions (see $\S 3$ for the details). It is known that

$$
\mathcal{C}=\overline{\mathcal{C}}^{(1)} \leq \cdots \leq \overline{\mathcal{C}}^{(n)}=\cdots=\overline{\mathcal{C}}^{(\infty)},
$$

where $\overline{\mathcal{C}}(m)$ is the $m$-closure of $\mathcal{C}$ (i.e., the restriction of the $m$-extension to the diagonal of $V^{m}$ identified with $\left.V\right), \overline{\mathcal{C}}^{(\infty)}$ is the scheme of the group $\operatorname{Aut}(\mathcal{C})$, and $n=|V|$, and that

$$
\operatorname{Sim}\left(\mathcal{C}, \mathcal{C}^{\prime}\right)=\operatorname{Sim}_{1}\left(\mathcal{C}, \mathcal{C}^{\prime}\right) \supset \cdots \supset \operatorname{Sim}_{n}\left(\mathcal{C}, \mathcal{C}^{\prime}\right)=\cdots=\operatorname{Sim}_{\infty}\left(\mathcal{C}, \mathcal{C}^{\prime}\right)
$$

where $\operatorname{Sim}_{m}\left(\mathcal{C}, \mathcal{C}^{\prime}\right)$ is the set of all $m$-similarities from $\mathcal{C}$ to $\mathcal{C}^{\prime}$ (i.e., the similarities admitting an $m$-extension) and $\operatorname{Sim}_{\infty}\left(\mathcal{C}, \mathcal{C}^{\prime}\right)$ is the set of all similarities from $\mathcal{C}$ to $\mathcal{C}^{\prime}$ induced by isomorphisms of these schemes. In fact, the starting point of this paper was to determine the 2-extension of the scheme of a projective plane and to find a sufficient condition for the latter scheme to be 2-closed. Generally, the problem of finding the $m$-extension of a scheme seems to be very hard. Apart from trivial cases, it was completely solved only for cyclotomic schemes over a finite field [2]. In our case the answer is given by the following statement.

Theorem 1.2. Let $\mathcal{C}$ be a scheme of a finite projective plane $\mathcal{P}$, and let $\widehat{\mathcal{C}}$ be its 2extension. Then:

1) $\widehat{\mathcal{C}}$ equals the derivative scheme of $\mathcal{P}$;

2) the scheme $\mathcal{C}$ is 2-closed.

Let $\mathcal{C}$ and $\mathcal{C}^{\prime}$ be schemes of finite projective planes $\mathcal{P}$ and $\mathcal{P}^{\prime}$ of the same order with the sets of basis relations $\mathcal{R}$ and $\mathcal{R}^{\prime}$, respectively. Then the bijection from $\mathcal{R}$ to $\mathcal{R}^{\prime}$ preserving the types of basis relations gives a similarity $\varphi: \mathcal{C} \rightarrow \mathcal{C}^{\prime}$ called the canonical one. By 
statement 2) of Theorem 1.1, we have $\operatorname{Der}(\mathcal{P})=\operatorname{Der}\left(\mathcal{P}^{\prime}\right)$. So the mapping $\hat{\varphi}$ from $\Pi$ to $\Pi^{\prime}$ such that

$$
R(\Gamma, \mathcal{P})^{\hat{\varphi}}=R\left(\Gamma, \mathcal{P}^{\prime}\right), \quad \Gamma \in \operatorname{Der}(\mathcal{P}),
$$

where $\Pi$ and $\Pi^{\prime}$ are the derivative partitions associated with $\mathcal{P}$ and $\mathcal{P}^{\prime}$, respectively, is a bijection. Thus, from statement 3) of Theorem 1.1 and statement 1) of Theorem 1.2 it follows that $\hat{\varphi}$ is a similarity from $\hat{\mathcal{C}}$ to $\hat{\mathcal{C}}^{\prime}$, which is obviously the 2-extension of $\varphi$. This proves the following result.

Theorem 1.3. The canonical similarity between the schemes of two finite projective planes of the same order is in fact a 2-similarity.

There are natural analogs of Theorems 1.1 and 1.3 for the homogeneous scheme of a projective plane $\mathcal{P}$ defined as follows. Let $\mathcal{C}$ be the nonhomogeneous scheme of the plane $\mathcal{P}$. Then it is easily seen that the group $\Phi=\operatorname{Sim}(\mathcal{C}, \mathcal{C})$ consists of two elements. Denote by $\varphi$ the nontrivial one. Clearly, $\varphi$ coincides with the canonical similarity from $\mathcal{C}$ to the scheme of the projective plane dual to $\mathcal{P}$. (So, by Theorem 1.3 , the similarity $\varphi$ has a 2-extension $\hat{\varphi}$.) The scheme $\mathcal{D}=\mathcal{C}^{\Phi}$ obtained from $\mathcal{C}$ by merging the relations in each $\Phi$-orbit is called the homogeneous scheme of $\mathcal{P}$. One can see that $\mathcal{D}$ is the scheme of the incidence graph of $\mathcal{P}$, which is a bipartite distance-regular graph of diameter 3. If $\mathcal{C}^{\prime}$ is the scheme of a projective plane $\mathcal{P}^{\prime}$ of the same order as $\mathcal{P}$, then the canonical similarity from $\mathcal{C}$ to $\mathcal{C}^{\prime}$ induces by restriction a similarity $\psi: \mathcal{D} \rightarrow \mathcal{D}^{\prime}$, where $\mathcal{D}^{\prime}$ is the homogeneous scheme of $\mathcal{P}^{\prime}$. Being, obviously, the only element of $\operatorname{Sim}\left(\mathcal{D}, \mathcal{D}^{\prime}\right)$, the similarity $\psi$ is called the canonical similarity from $\mathcal{D}$ to $\mathcal{D}^{\prime}$.

Theorem 1.4. In the above notation we have:

1) $\widehat{\mathcal{D}}=\widehat{\mathcal{C}}^{\widehat{\Phi}}$, where $\widehat{\Phi}=\left\{1_{\hat{\mathcal{C}}}, \widehat{\varphi}\right\}$;

2) the scheme $\mathcal{D}$ is 2 -closed;

3) the canonical similarity from $\mathcal{D}$ to the homogeneous scheme of another projective plane of the same order is in fact a 2-similarity.

In the study of permutation groups of rank 3, for strongly regular graphs Higman [1] introduced the notion of a $t$-condition, which was generalized to arbitrary colored graphs in [9] (see also 6]). Below, by the colored graph of a scheme $(V, \mathcal{R})$ we mean a graph, the vertex set and the set of colored classes of which are $V$ and $\mathcal{R}$, respectively. By $[\underline{6}$, Theorem 6.4], the colored graph of any 2-closed scheme satisfies the 6-condition. So, statement 2) of Theorem 1.4 implies the following result, which shows in particular that there are infinitely many distance-regular graphs of diameter 3 that satisfy the 6 -condition and are not distance-transitive.

Corollary 1.5. The colored graph of the scheme (homogeneous or not) of any finite projective plane satisfies the 6-condition.

In the framework of the theory of $m$-extensions, the most important invariants of a scheme $\mathcal{C}$ are the Schurity number $t(\mathcal{C})$ and the separability number $s(\mathcal{C})$ introduced in 66 with the help of inclusions (3) and (4), respectively (see \$3). Let us analyze these invariants in the case where $\mathcal{C}$ is the (homogeneous or not) scheme of a projective plane $\mathcal{P}$ of order $q$. We observe that, by the Ostrom-Wagner theorem [14, Theorem 14.13], the plane $\mathcal{P}$ is a Galois plane if and only if its group of collineations acts 2-transitively on the points of $\mathcal{P}$. Thus, $t(\mathcal{C})=1$ if and only if $\mathcal{P}$ is a Galois plane (cf. [6, Theorem 7.9]). Moreover, it is easily seen that $s(\mathcal{C})=1$ if and only if any projective plane of order $q$ is isomorphic to $\mathcal{P}$ in the nonhomogeneous case, and either to $\mathcal{P}$ or to its dual plane in the homogeneous case.

Next, the definitions show that if $t(\mathcal{C})=2$ (respectively, $s(\mathcal{C})=2$ ), then $\mathcal{C}<\overline{\mathcal{C}}(\infty)=$ $\overline{\mathcal{C}}^{(2)}$ (respectively, $\operatorname{Sim}\left(\mathcal{C}, \mathcal{C}^{\prime}\right) \supseteqq \operatorname{Sim}_{\infty}\left(\mathcal{C}, \mathcal{C}^{\prime}\right)=\operatorname{Sim}_{2}\left(\mathcal{C}, \mathcal{C}^{\prime}\right)$ for some scheme $\left.\mathcal{C}^{\prime}\right)$. However, 
if $\mathcal{C}$ is the nonhomogeneous scheme of a projective plane, this contradicts statement 2) of Theorem 1.2 (respectively, statement 2) of Theorem 1.3), whereas if $\mathcal{C}$ is homogeneous, this contradicts statement 2) of Theorem 1.4 (respectively, statement 3) of Theorem 1.4). Thus, we arrive at the following result.

Corollary 1.6. The Schurity and separability numbers of the scheme (homogeneous or not) of a projective plane cannot equal 2.

In [6, Theorem 7.9] it was proved that $s(\mathcal{C}) \leq 6$ whenever $\mathcal{C}$ is a homogeneous scheme of a Galois plane of order $q$. The following theorem generalizes and refines this result. From Corollary [1.6 it follows that the new upper bound is attained whenever there exists a non-Galois plane of order $q$ (there are infinitely many such numbers $q$; see [14]).

Theorem 1.7. Let $\mathcal{C}$ be the scheme (homogeneous or not) of a Galois plane. Then $s(\mathcal{C}) \leq 3$.

The proof of Theorem 1.1 occupies $\S \$ 4$ In the first two of those sections, we introduce the concept of a configuration (generalizing that of a $\Xi$-configuration) and study general properties of configurations as well as their embeddings in projective planes 2 The proof itself is in $₫ \sqrt{6}$. The key point is to show that the numbers of embeddings of special configurations in a projective plane of order $q$ depend only on $q$; these numbers are in fact the intersection numbers of the derivative scheme. The configurations in question turn out to be admissible in the sense of 97 (Theorem 7.3 ). In 88 we prove that the embedding number of any admissible configuration can be expressed via those of admissible configurations of smaller size (Theorem 7.4). The proofs of Theorems 1.2, 1.4. and 1.7 are contained in $\S \$ 9$, 10, and 11, respectively. In the Appendix, for any projective plane we present the list of $\Xi$-configurations parameterizing the basis relations of the derivative scheme. To make the paper self-contained as far as possible, we cite the background on schemes and their multidimensional extensions in $\S \S 2$ and 3 (the details can be found in [1]).

Part of the results related to homogeneous schemes of projective planes was announced in [3. Concerning the theory of finite projective planes we refer the reader to [14].

Notation. As usual, we denote by $\mathbb{Z}$ and $\mathbb{R}$ the ring of integers and the field of reals, respectively.

The cardinality of a finite set $V$ is denoted by $|V|$, and the diagonal of $V^{2}$ by $\Delta(V)$. For a (binary) relation $R$ on $V$ and $v \in V$, we set

$$
\begin{gathered}
R^{T}=\left\{(u, v) \in V^{2}:(v, u) \in R\right\}, \\
R_{\text {in }}(v)=\{u \in V:(u, v) \in R\}, \quad R_{\text {out }}(v)=\{u \in V:(v, u) \in R\} .
\end{gathered}
$$

For relations $R, S \subset V^{2}$, we set

$$
\begin{aligned}
R \cdot S & =\left\{(u, w) \in V^{2}:(u, v) \in R,(v, w) \in S \text { for some } v \in V\right\}, \\
R \otimes S & =\left\{\left(\left(u_{1}, u_{2}\right),\left(v_{1}, v_{2}\right)\right) \in\left(V^{2}\right)^{2}:\left(u_{1}, v_{1}\right) \in R,\left(u_{2}, v_{2}\right) \in S\right\} .
\end{aligned}
$$

For an equivalence relation $E$ on $V$, we denote by $V / E$ the set of its classes. If $R$ is a relation on $V$, then we set

$$
R_{V / E}=\left\{(X, Y) \in(V / E)^{2}: R_{X, Y} \neq \emptyset\right\}
$$

where $R_{X, Y}=R \cap(X \times Y)$. For $X \subset V$ the set $R_{X}=R_{X, X}$ is treated as a relation on $X$.

The group of all permutations of $V$ is denoted by $\operatorname{Sym}(V)$.

${ }^{2}$ The idea to use configurations was inspired by the paper 10 . 
Each bijection $f: V \rightarrow V^{\prime}\left(v \mapsto v^{f}\right)$ determines naturally a bijection $R \mapsto R^{f}$ from the relations on $V$ onto the relations on $V^{\prime}$ and a group isomorphism $g \mapsto g^{f}$ from $\operatorname{Sym}(V)$ onto $\operatorname{Sym}\left(V^{\prime}\right)$. For an equivalence relation $E$ on $V$, the bijection $f$ induces a bijection $f_{V / E}: V / E \rightarrow V^{\prime} / E^{\prime}$, where $E^{\prime}=E^{f}$.

For $i=1, \ldots, m$, the $i$ th coordinate of $x \in V^{m}$ is denoted by $x_{i}$.

The set of elements (points and lines) of a projective plane $\mathcal{P}$ is denoted by $V$.

To define the type of an element of $\mathcal{P}$, we introduce the two-element set $\mathfrak{T}_{1}=\{\mathfrak{p}, \mathfrak{l}\}$ and a function $t_{1, \mathcal{P}}: V \rightarrow \mathfrak{T}_{1}$. By definition, $t_{1, \mathcal{P}}(x)=\mathfrak{p}$ (respectively, $t_{1, \mathcal{P}}(x)=\mathfrak{l}$ ) if and only if $x$ is a point (respectively, line) of the plane $\mathcal{P}$. The elements $\mathfrak{p}$ and $\mathfrak{l}$ are said to be dual to each other.

To define the type of a relation between two elements of $\mathcal{P}$, we introduce the fourelement set $\mathfrak{T}_{2}=\left\{\mathfrak{e}, \mathfrak{e}^{\prime}, \mathfrak{i}, \mathfrak{i}^{\prime}\right\}$ and a function $t_{2, \mathcal{P}}: V^{2} \rightarrow \mathfrak{T}_{2}$. By definition $t_{2, \mathcal{P}}(x, y)=\mathfrak{e}$ (respectively, $t_{2, \mathcal{P}}(x, y)=\mathfrak{e}^{\prime}$ ) if and only if $x, y$ are equal (respectively, unequal) elements of the same type; similarly, $t_{2, \mathcal{P}}(x, y)=\mathfrak{i}$ (respectively, $\left.t_{2, \mathcal{P}}(x, y)=\mathfrak{i}^{\prime}\right)$ if and only if $x, y$ are incident (respectively, nonincident) elements of distinct types.

When it does not lead to misunderstanding, we omit the subscript $\mathcal{P}$ in $t_{1, \mathcal{P}}$ and $t_{2, \mathcal{P}}$.

For distinct elements $x, y$ of $\mathcal{P}$ of the same type, the (only) element incident to both of them is denoted by $x y$.

\section{$\S 2$. SCHEMES AND SIMILARITIES}

Let $V$ be a finite set and $\mathcal{R}$ a partition of $V^{2}$ closed with respect to transposition. Denote by $\mathcal{R}^{*}$ the set of all unions of elements of $\mathcal{R}$. A pair $\mathcal{C}=(V, \mathcal{R})$ is called a coherent configuration or a scheme on $V$ if the diagonal $\Delta(V)$ of $V^{2}$ belongs to $\mathcal{R}^{*}$ and if, for any $R, S, T \in \mathcal{R}$, the number

$$
c_{R, S}(u, w)=|\{v \in V:(u, v) \in R,(v, w) \in S\}|
$$

does not depend on the choice of $(u, w) \in T$. The elements of the sets $V, \mathcal{R}=\mathcal{R}(\mathcal{C})$, $\mathcal{R}^{*}=\mathcal{R}^{*}(\mathcal{C})$, and the numbers (5) are called the points, the basis relations, the relations and the intersection numbers of $\mathcal{C}$, respectively; the intersection numbers are denoted by $c_{R, S}^{T}$. From the definition it follows that $R \cdot S \in \mathcal{R}^{*}$ for all $R, S \in \mathcal{R}^{*}$. The number $\operatorname{rk}(\mathcal{C})=|\mathcal{R}|$ is called the rank of $\mathcal{C}$.

The set $V$ is the disjoint union of homogeneity sets of $\mathcal{C}$, i.e., of sets $X \subset V$ for which $\Delta(X) \in \mathcal{R}$. For any $R \in \mathcal{R}$, two homogeneity sets $X$ and $Y$ such that $R \subset X \times Y$ exist and are uniquely determined. Put $d_{\text {out }}(R)=c_{R, R^{T}}^{\Delta(X)}$ and $d_{\text {in }}(R)=c_{R^{T}, R}^{\Delta(Y)}$. Then

$$
d_{\text {out }}(R)=\left|R_{\text {out }}(x)\right|, \quad d_{\text {in }}(R)=\left|R_{\text {in }}(y)\right|, \quad x \in X, y \in Y .
$$

By a set of $\mathcal{C}$ we mean any union of homogeneity sets. It is easily seen that $X$ and $Y$ are sets of $\mathcal{C}$ if and only if $X \times Y \in \mathcal{R}^{*}$. The scheme $\mathcal{C}$ is called homogeneous if $V$ is a homogeneity set of it.

We say that two schemes are isomorphic if there exists a bijection between their point sets preserving the basis relations. Any such bijection is called an isomorphism of these schemes. The group of all isomorphisms of a scheme $\mathcal{C}$ contains a normal subgroup

$$
\operatorname{Aut}(\mathcal{C})=\left\{f \in \operatorname{Sym}(V): R^{f}=R, R \in \mathcal{R}\right\}
$$

called the automorphism group of $\mathcal{C}$. On the other hand, let $G \leq \operatorname{Sym}(V)$ be a permutation group and $\mathcal{R}$ the set of orbits of the coordinatewise action of $G$ on $V^{2}$. Then $(V, \mathcal{R})$ is a scheme; we call it the scheme of $G$.

Given a set $X \subset V$, we denote by $\mathcal{R}_{X}$ the set of all nonempty relations $R_{X}, R \in \mathcal{R}$. If $X$ is a set of $\mathcal{C}$, then $\mathcal{C}_{X}=\left(X, \mathcal{R}_{X}\right)$ is a scheme. Given an equivalence relation $E$ on $V$, we denote by $\mathcal{R}_{V / E}$ the set of all nonempty relations $R_{V / E}, R \in \mathcal{R}$. If $E \in \mathcal{R}^{*}$, then 
$\mathcal{C}_{V / E}=\left(V / E, \mathcal{R}_{V / E}\right)$ is a scheme. The set of all equivalence relations $E \in \mathcal{R}^{*}$ is denoted by $\mathcal{E}$.

For schemes $\mathcal{C}_{1}=\left(V_{1}, \mathcal{R}_{1}\right)$ and $\mathcal{C}_{2}=\left(V_{2}, \mathcal{R}_{2}\right)$, let $\mathcal{R}_{1} \otimes \mathcal{R}_{2}$ denote the set of all relations $R_{1} \otimes R_{2}, R_{1} \in \mathcal{R}_{1}, R_{2} \in \mathcal{R}_{2}$. Then the pair $\mathcal{C}_{1} \otimes \mathcal{C}_{2}=\left(V_{1} \times V_{2}, \mathcal{R}_{1} \otimes \mathcal{R}_{2}\right)$ is a scheme. It is called the tensor product of $\mathcal{C}_{1}$ and $\mathcal{C}_{2}$.

The set of all schemes on $V$ is partially ordered by inclusion, namely, $\mathcal{C} \leq \mathcal{C}^{\prime}$ if and only if $\mathcal{R}^{*} \subset\left(\mathcal{R}^{\prime}\right)^{*}$; in this case, we call $\mathcal{C}^{\prime}$ an extension of $\mathcal{C}$. For sets $\mathcal{R}_{1}, \ldots, \mathcal{R}_{s}$ of binary relations on $V$, we denote by $\left[\mathcal{R}_{1}, \ldots, \mathcal{R}_{s}\right]$ the smallest scheme $\mathcal{C}$ on $V$ such that $\mathcal{R}_{i} \subset \mathcal{R}^{*}$ for all $i$; we omit the square brackets if $\mathcal{R}_{i}=\left\{R_{i}\right\}$ and write $\mathcal{C}_{i}$ instead of $\mathcal{R}_{i}$ if the latter is the set of basis relations of $\mathcal{C}_{i}$. For an equivalence relation $E$ on $V$ we set $\mathcal{C}_{E}=[\mathcal{C},\{\Delta(X): X \in V / E\}]$

Schemes $\mathcal{C}$ and $\mathcal{C}^{\prime}$ are said to be similar if

$$
c_{R, S}^{T}=c_{R^{\varphi}, S^{\varphi}}^{T^{\varphi}}, \quad R, S, T \in \mathcal{R},
$$

for some bijection $\varphi: \mathcal{R} \rightarrow \mathcal{R}^{\prime}, R \mapsto R^{\varphi}$, called a similarity from $\mathcal{C}$ to $\mathcal{C}^{\prime}$. The set of all similarities is denoted by $\operatorname{Sim}\left(\mathcal{C}, \mathcal{C}^{\prime}\right)$. In a natural way, each isomorphism from $\mathcal{C}$ to $\mathcal{C}^{\prime}$ induces a similarity between these schemes. The set of all isomorphisms from $\mathcal{C}$ to $\mathcal{C}^{\prime}$ inducing a similarity $\varphi$ is denoted by $\operatorname{Iso}\left(\mathcal{C}, \mathcal{C}^{\prime}, \varphi\right)$.

A similarity $\varphi$ induces bijections both between the sets and between the relations of $\mathcal{C}$ and $\mathcal{C}^{\prime}$; we use the same letter $\varphi$ to denote these bijections. It can be seen that $\mathcal{E}^{\varphi}=\mathcal{E}^{\prime}$, $V^{\varphi}=V^{\prime}$, and

$$
\left(R^{T}\right)^{\varphi}=\left(R^{\varphi}\right)^{T}, \quad d_{\text {out }}(R)=d_{\text {out }}\left(R^{\varphi}\right), \quad d_{\text {in }}(R)=d_{\text {in }}\left(R^{\varphi}\right), \quad R \in \mathcal{R} .
$$

For any $E \in \mathcal{E}$, the similarity $\varphi$ induces a similarity $\varphi_{V / E}: \mathcal{C}_{V / E} \rightarrow \mathcal{C}_{V^{\prime} / E^{\prime}}^{\prime}$, where $E^{\prime}=E^{\varphi}$. Finally, for a group $\Phi \leq \operatorname{Sim}(\mathcal{C}, \mathcal{C})$, we set

$$
\mathcal{R}^{\Phi}=\left\{R^{\Phi}: R \in \mathcal{R}\right\},
$$

where $R^{\Phi}=\bigcup_{\varphi \in \Phi} R^{\varphi}$. Then the pair $\mathcal{C}^{\Phi}=\left(V, \mathcal{R}^{\Phi}\right)$ is a scheme.

A scheme $\mathcal{C}=(V, \mathcal{R})$ is semiregular if $d_{\text {out }}(R) \leq 1$ and $d_{\text {in }}(R) \leq 1$ for all $R \in \mathcal{R}$. For homogeneous schemes, the first three statements of the following theorem were proved in [15].

Theorem 2.1. Let $\mathcal{C}=(V, \mathcal{R})$ be a scheme, and let $E \in \mathcal{E}$. Suppose that the scheme $\mathcal{C}_{V / E}$ is semiregular. Then:

1) $\mathcal{R}\left(\mathcal{C}_{E}\right)=\left\{R_{X, Y}: R \in \mathcal{R}, X, Y \in V / E, R_{X, Y} \neq \varnothing\right\}$;

2) for every $g \in \operatorname{Aut}\left(\mathcal{C}_{V / E}\right)$, the mapping $\psi_{g}: R_{X, Y} \mapsto R_{X^{g}, Y^{g}}$ is a similarity of $\mathcal{C}_{E}$

3) $\mathcal{C}=\left(\mathcal{C}_{E}\right)^{\Psi}$, where $\Psi=\left\{\psi_{g}: g \in \operatorname{Aut}\left(\mathcal{C}_{V / E}\right)\right\}$;

4) for any similarity $\varphi: \mathcal{C} \rightarrow \mathcal{C}^{\prime}$ there exists a similarity $\varphi_{E}: \mathcal{C}_{E} \rightarrow \mathcal{C}_{E^{\prime}}^{\prime}$ extending $\varphi$, where $E^{\prime}=E^{\varphi} ;$ moreover, for each $f \in \operatorname{Iso}\left(\mathcal{C}_{V / E}, \mathcal{C}_{V^{\prime} / E^{\prime}}^{\prime}, \varphi_{V / E}\right)$ the similarity $\varphi_{E}$ can be chosen so that $\left(\varphi_{E}\right)_{V / E}$ is induced by $f$.

Proof. First, we prove that, given $R, S, T \in \mathcal{R}$ and $X, X^{\prime}, X^{\prime \prime}, Y, Y^{\prime}, Y^{\prime \prime} \in V / E$ with $R_{X, Y} \neq \varnothing$ and $S_{X^{\prime}, Y^{\prime}} \neq \varnothing$, we have

$$
c_{R_{X, Y}, S_{X^{\prime}, Y^{\prime}}}(u, v)=\delta_{X^{\prime \prime}, X} \delta_{Y, X^{\prime}} \delta_{Y^{\prime}, Y^{\prime \prime}} c_{R, S}^{T}, \quad(u, v) \in T_{X^{\prime \prime}, Y^{\prime \prime}} .
$$

Without loss of generality we assume that $X^{\prime \prime}=X, Y=X^{\prime}$, and $Y^{\prime}=Y^{\prime \prime}$. Since $R_{X, Y} \neq \varnothing$ and $S_{X^{\prime}, Y^{\prime}} \neq \varnothing$, the semiregularity of $\mathcal{C}_{V / E}$ implies that $\left(R_{V / E}\right)_{\text {out }}(X)=\{Y\}$ and $\left(S_{V / E}\right)_{\text {in }}\left(Y^{\prime}\right)=\left\{X^{\prime}\right\}$ for some $X^{\prime}, Y \in V / E$. So,

$$
c_{R, S}(u, v)=c_{R_{X, Y}, S_{X^{\prime}, Y^{\prime}}}(u, v), \quad(u, v) \in X \times Y^{\prime} .
$$

If $(u, v) \in T$, then the left-hand side equals $c_{R, S}^{T}$, and (8) follows. 
Denote by $\mathcal{R}^{\prime}$ the right-hand side in statement 1$)$ of the theorem. Clearly, $\mathcal{R}^{\prime}$ is a partition of $V^{2}$ closed with respect to transposition, and $\Delta(V) \in\left(\mathcal{R}^{\prime}\right)^{*}$. Therefore, (8) implies that $\mathcal{C}^{\prime}=\left(V, \mathcal{R}^{\prime}\right)$ is a scheme, the intersection numbers of which look like this:

$$
c_{R_{X, Y}, S_{X^{\prime}, Y^{\prime}}}^{T_{X^{\prime \prime}, Y^{\prime \prime}}}=\delta_{X^{\prime \prime}, X} \delta_{Y, X^{\prime}} \delta_{Y^{\prime}, Y^{\prime \prime}} c_{R, S}^{T} .
$$

Since, obviously, $\mathcal{C}^{\prime} \geq \mathcal{C}$ and $\mathcal{C}_{E} \geq \mathcal{C}^{\prime}$, we conclude that $\mathcal{C}^{\prime}=\mathcal{C}_{E}$. Statement 1) is proved. Statement 2) follows immediately from (9) and the definition of $\psi_{g}$. Finally, by [6. Theorem 4.4], the automorphism group of a semiregular scheme acts transitively on any of its basis relations. Thus, given $R \in \mathcal{R}$, we have

$$
R=\bigcup_{(X, Y) \in R_{V / E}} R_{X, Y}=\bigcup_{g \in \operatorname{Aut}\left(\mathcal{C}_{V / E}\right)} R_{X_{0}^{g}, Y_{0}^{g}},
$$

where $X_{0}, Y_{0} \in V / E$ with $R_{X_{0}, Y_{0}} \neq \varnothing$. This proves statement 3$)$.

To prove statement 4 ), we let $\varphi: \mathcal{C} \rightarrow \mathcal{C}^{\prime}$ be a similarity. Then $\mathcal{C}_{V^{\prime} / E^{\prime}}^{\prime}$ coincides with the $\varphi_{V / E}$-image of $\mathcal{C}_{V / E}$ and hence it is semiregular (see (7)). Therefore, $\operatorname{Iso}\left(\mathcal{C}_{V / E}, \mathcal{C}_{V^{\prime} / E^{\prime}}^{\prime}, \varphi_{V / E}\right) \neq \varnothing$ by [6, Theorem 4.4]. Take $f \in \operatorname{Iso}\left(\mathcal{C}_{V / E}, \mathcal{C}_{V^{\prime} / E^{\prime}}^{\prime}, \varphi_{V / E}\right)$. By (9), the mapping $R_{X, Y} \mapsto R_{X^{f}, Y^{f}}^{\prime}, R_{X, Y} \in \mathcal{R}\left(\mathcal{C}_{E}\right)$ induces a similarity from $\mathcal{C}_{E}$ to $\mathcal{C}_{E^{\prime}}^{\prime}$ Since the restriction of it to $\mathcal{C}$ coincides with $\varphi$, we are done.

Let $\mathcal{P}$ be a projective plane of order $q, V_{\mathfrak{p}}=t_{1, \mathcal{P}}^{-1}(\mathfrak{p})$ and $V_{\mathfrak{l}}=t_{1, \mathcal{P}}^{-1}(\mathfrak{l})$ the sets of its points and lines, $V=V_{\mathfrak{p}} \cup V_{\mathfrak{l}}$, and $\mathcal{R}$ the set of the following eight relations (cf. (1) and (2)):

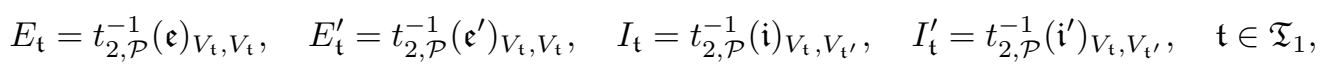

where $\mathfrak{t}^{\prime}$ is the dual to $\mathfrak{t}$ (see the notation). Then it is easily seen that the pair $\mathcal{C}=(V, \mathcal{R})$ is a scheme on $2\left(q^{2}+q+1\right)$ points; it is called the (nonhomogeneous) scheme of $\mathcal{P}$. This scheme has two homogeneity sets $V_{\mathfrak{p}}$ and $V_{\mathfrak{l}}$ and satisfies the following conditions with $X=V_{\mathfrak{p}}, Y=V_{\mathfrak{l}}$ and $R=I_{\mathfrak{p}}$, or $X=V_{\mathfrak{l}}, Y=V_{\mathfrak{p}}$ and $R=I_{\mathfrak{l}}$ :

(P1) $\operatorname{rk}(\mathcal{C})=8$ and $\operatorname{rk}\left(\mathcal{C}_{X}\right)=\operatorname{rk}\left(\mathcal{C}_{Y}\right)=2$,

(P2) $c_{R, R^{T}}^{S}=1$ for some basis relation $R \subset X \times Y$ and $S=X^{2} \backslash \Delta(X)$.

In fact, any scheme $\mathcal{C}$ with two homogeneity sets $X$ and $Y$ satisfying (P1) and (P2) is the scheme of some projective plane. We note that the degrees $\left(d_{\text {in }}\right.$ and $\left.d_{\text {out }}\right)$ of the relations $E_{\mathfrak{t}}, E_{\mathfrak{t}}^{\prime}, I_{\mathfrak{t}}$, and $I_{\mathfrak{t}}^{\prime}$ equal $1, q^{2}+q, q+1$, and $q^{2}$ (respectively), where $\mathfrak{t} \in\{\mathfrak{p}, \mathfrak{l}\}$.

Let $\varphi: \mathcal{C} \rightarrow \mathcal{C}^{\prime}$ be a similarity. From what has been said above and the properties of similarities, we show that $\mathcal{C}^{\prime}$ is also the scheme of a projective plane of order $q$. Moreover, $\varphi$ takes the basis relations of $\mathcal{C}$ either to the corresponding basis relations of $\mathcal{C}^{\prime}$, or to the dual ones 3 Conversely, any of these two mappings induces a similarity from $\mathcal{C}$ to $\mathcal{C}^{\prime}$ (the first of them is said to be canonical). In particular, $\Phi=\operatorname{Sim}(\mathcal{C}, \mathcal{C})$ is a group of order 2. The scheme $\mathcal{D}=\mathcal{C}^{\Phi}$ is called the homogeneous scheme of $\mathcal{P}$. As above, any scheme similar to $\mathcal{D}$ is the homogeneous scheme of a projective plane of the same order. Moreover, for any two homogeneous schemes of projective planes of the same order, there exists a unique similarity between them called canonical.

\section{§3. EXTENSIONS OF SCHEMES AND SIMILARITIES}

Let $\mathcal{C}$ be a scheme on $V$ and $m$ a positive integer. By definition, the $m$-extension of $\mathcal{C}$ is the following scheme on $V^{m}$ :

$$
\widehat{\mathcal{C}}^{(m)}=\left[\mathcal{C}^{m}, \Delta_{m}\right]
$$

\footnotetext{
${ }^{3}$ The relations $E_{\mathfrak{p}}$ and $E_{\mathfrak{l}}, E_{\mathfrak{p}}^{\prime}$ and $E_{\mathfrak{l}}^{\prime}, I_{\mathfrak{p}}$ and $I_{\mathfrak{l}}, I_{\mathfrak{p}}^{\prime}$ and $I_{\mathfrak{l}}^{\prime}$ are said to be dual to each other.
} 
where $\mathcal{C}^{m}$ is the $m$-fold tensor product of $\mathcal{C}$ and $\Delta_{m}$ is the diagonal of $V^{m}$. We observe that, except for trivial cases, the $m$-extension is a nonhomogeneous scheme for all $m \geq 2$. The above definition implies that $\hat{\mathcal{C}}^{(1)}=\mathcal{C}$ and $\hat{\mathcal{C}}^{(m)}$ is a fusion of the scheme corresponding to the coordinatewise action of the group $\operatorname{Aut}(\mathcal{C})$ on $V^{m}$. Moreover,

$$
\operatorname{Aut}(\mathcal{C}) \cong \operatorname{Aut}\left(\hat{\mathcal{C}}^{(m)}\right)_{\Delta_{m}} \cong \operatorname{Aut}\left(\widehat{\mathcal{C}}^{(m)}\right) .
$$

For any $m$ the intersection numbers of the $m$-extension of $\mathcal{C}$ are invariants of it. Namely, a similarity $\psi: \hat{\mathcal{C}}^{(m)} \rightarrow \widehat{\mathcal{C}}^{(m)}$ is called an $m$-extension of a similarity $\varphi: \mathcal{C} \rightarrow \mathcal{C}^{\prime}$ if

1) $\left(\Delta_{m}\right)^{\psi}=\Delta_{m}^{\prime}$,

2) $R^{\psi}=R^{\varphi^{m}}$ for all $R \in \mathcal{R}\left(\mathcal{C}^{m}\right)$,

where $\varphi^{m}: \mathcal{C}^{m} \rightarrow\left(\mathcal{C}^{\prime}\right)^{m}$ is the $m$-fold tensor product of $\varphi$. This definition shows that each similarity has a 1-extension coinciding with it. Moreover, for any $m$, the existence of an $m$-extension of $\varphi$ implies its uniqueness; we denote it by $\hat{\varphi}^{(m)}$. Observe that not every similarity admits an $m$-extension. However, if $\varphi$ is induced by some isomorphism, then $\varphi$ has an $m$-extension for all $m$.

Set

$$
\overline{\mathcal{C}}=\overline{\mathcal{C}}^{(m)}=\left(\left(\widehat{\mathcal{C}}^{(m)}\right)_{\Delta}\right)^{\delta^{-1}}
$$

where $\delta: v \mapsto(v, \ldots, v)$ is the diagonal mapping from $V$ to $V^{m}$. The scheme $\overline{\mathcal{C}}$ is called the $m$-closure of $\mathcal{C}$; if $\mathcal{C}=\overline{\mathcal{C}}^{(m)}$, then we say that $\mathcal{C}$ is $m$-closed. A similarity between two schemes is called an $m$-similarity if it admits an $m$-extension. Any such similarity $\varphi$ is extended uniquely to a similarity $\bar{\varphi}^{(m)}$ between the $m$-closures. The set of all $m$-similarities from $\mathcal{C}$ to $\mathcal{C}^{\prime}$ is denoted by $\operatorname{Sim}_{m}\left(\mathcal{C}, \mathcal{C}^{\prime}\right)$. The following theorem was proved in [5].

Theorem 3.1. For an arbitrary scheme $\mathcal{C}$ on $n$ points, we have

$$
\begin{aligned}
\mathcal{C}=\overline{\mathcal{C}}^{(1)} & \leq \cdots \leq \overline{\mathcal{C}}^{(n)}=\cdots=\overline{\mathcal{C}}^{(\infty)}, \\
\operatorname{Sim}\left(\mathcal{C}, \mathcal{C}^{\prime}\right)=\operatorname{Sim}_{1}\left(\mathcal{C}, \mathcal{C}^{\prime}\right) & \supset \cdots \supset \operatorname{Sim}_{n}\left(\mathcal{C}, \mathcal{C}^{\prime}\right)=\cdots=\operatorname{Sim}_{\infty}\left(\mathcal{C}, \mathcal{C}^{\prime}\right)
\end{aligned}
$$

where $\overline{\mathcal{C}}^{(\infty)}$ is the scheme of the group $\operatorname{Aut}(\mathcal{C})$ and $\operatorname{Sim}_{\infty}\left(\mathcal{C}, \mathcal{C}^{\prime}\right)$ is the set of all similarities from $\mathcal{C}$ to $\mathcal{C}^{\prime}$ induced by isomorphisms of these schemes.

A scheme $\mathcal{C}$ is said to be $m$-Schurian if $\overline{\mathcal{C}}^{(m)}=\overline{\mathcal{C}}^{(\infty)}$ and $m$-separable if $\operatorname{Sim}_{m}\left(\mathcal{C}, \mathcal{C}^{\prime}\right)=$ $\operatorname{Sim}_{\infty}\left(\mathcal{C}, \mathcal{C}^{\prime}\right)$ for all $\mathcal{C}^{\prime}$. The integers

$$
t(\mathcal{C})=\min \{m: \mathcal{C} \text { is } m \text {-Schurian }\}, \quad s(\mathcal{C})=\min \{m: \mathcal{C} \text { is } m \text {-separable }\}
$$

are called the Schurity number and the separability number of $\mathcal{C}$, respectively. It can be checked that $t(\mathcal{C})=s(\mathcal{C})=1$ whenever the scheme $\mathcal{C}$ is semiregular; see [6, Theorem 4.4].

The following theorem will be used throughout the paper; it is a straightforward consequence of [6, Lemma 6.2]. Below, given $R \subset V^{2}$ and $i, j \in\{1, \ldots, 2 m\}$, we set

$$
\mathrm{Cyl}_{R}(i, j)=\left\{x \in V^{2 m}:\left(x_{i}, x_{j}\right) \in R\right\} .
$$

Theorem 3.2. Let $\mathcal{C}$ be a scheme on $V$, and let $\widehat{\mathcal{C}}=\widehat{\mathcal{C}}^{(m)}$ and $\overline{\mathcal{C}}=\overline{\mathcal{C}}^{(m)}$, where $m$ is a positive integer. Then for any relation $R$ of the scheme $\overline{\mathcal{C}}$ and any $i, j \in\{1, \ldots, 2 m\}$, the following statements are true:

1) the set $\mathrm{Cyl}_{R}(i, j)$ is a relation of the scheme $\widehat{\mathcal{C}}$;

2) $\mathrm{Cyl}_{R}(i, j)^{\hat{\varphi}}=\mathrm{Cyl}_{R^{\bar{\varphi}}}(i, j)$ for any m-similarity $\varphi: \mathcal{C} \rightarrow \mathcal{C}^{\prime}$, where $\hat{\varphi}=\hat{\varphi}^{(m)}$ and $\bar{\varphi}=\bar{\varphi}^{(m)}$. 


\section{§4. Configurations}

Let $\Omega$ be a finite set. We shall consider colored undirected graphs on $\Omega$ with loops; the vertices and the edges of these graphs will be labeled with elements of the sets $\mathfrak{T}_{1}$ and $\mathfrak{T}_{2}$, respectively (see the notation). The edge set is identified with a symmetric binary relation $E$ on $\Omega$ such that $\Delta(\Omega) \subset E \subset \Omega^{2}$. The coloring is given by the functions

$$
t_{1}: \Omega \rightarrow \mathfrak{T}_{1}, \quad t_{2}: E \rightarrow \mathfrak{T}_{2} .
$$

Thus, such a graph $\Gamma$ is represented by the triple $(\Omega, E, t)$, where $t=\left(t_{1}, t_{2}\right)$.

Definition 4.1. A colored graph $\Gamma=(\Omega, E, t)$ is called a configuration on $\Omega$ if $\Delta(\Omega) \subset E_{\mathfrak{e}}$ and

$$
E_{\mathfrak{e}} \cup E_{\mathfrak{e}^{\prime}} \subset\left(\Omega_{\mathfrak{p}} \times \Omega_{\mathfrak{p}}\right) \cup\left(\Omega_{\mathfrak{l}} \times \Omega_{\mathfrak{l}}\right), \quad E_{\mathfrak{i}} \cup E_{\mathfrak{i}^{\prime}} \subset\left(\Omega_{\mathfrak{p}} \times \Omega_{\mathfrak{l}}\right) \cup\left(\Omega_{\mathfrak{l}} \times \Omega_{\mathfrak{p}}\right),
$$

where $\Omega_{\mathfrak{t}}=t_{1}^{-1}(\mathfrak{t})$ for $\mathfrak{t} \in \mathfrak{T}_{1}$ and $E_{\mathfrak{t}}=t_{2}^{-1}(\mathfrak{t})$ for $\mathfrak{t} \in \mathfrak{T}_{2}$.

We say that a configuration $\Gamma$ is complete if $E=\Omega^{2}$. Given a set $\Omega^{\prime} \subset \Omega$, the configuration obtained by restriction of $\Gamma$ to $\Omega^{\prime}$ is denoted by $\Gamma_{\Omega^{\prime}}$.

Let $\mathcal{P}$ be a finite projective plane. Then there is a natural complete configuration on the set $V$ of its elements, where $t_{1}=t_{1, \mathcal{P}}$ and $t_{2}=t_{2, \mathcal{P}}$ (see the notation). Let $i_{0}: \Omega_{0} \rightarrow V$ be a mapping, where $\Omega_{0} \subset \Omega$.

Definition 4.2. A mapping $i: \Omega \rightarrow V$ is called a strict $\Gamma$-extension of $i_{0}$ if $\left.i\right|_{\Omega_{0}}=i_{0}$ and

$$
t_{1}(\xi)=t_{1, \mathcal{P}}(i(\xi)), \quad \xi \in \Omega, \quad \text { and } \quad t_{2}(\xi, \eta)=t_{2, \mathcal{P}}(i(\xi), i(\eta)), \quad(\xi, \eta) \in E .
$$

If the latter identity holds true only if $t_{2}(\xi, \eta) \in\{\mathfrak{e}, \mathfrak{i}\}$, then the mapping $i$ is called a nonstrict $\Gamma$-extension.

The set and the number of all strict $\Gamma$-extensions (respectively, nonstrict $\Gamma$-extensions) of a mapping $i_{0}: \Omega_{0} \rightarrow V$ are denoted by $\operatorname{Emb}_{\text {str }}\left(\Gamma, i_{0}, \mathcal{P}\right)$ and $\mathrm{e}_{\mathrm{str}}\left(\Gamma, i_{0}, \mathcal{P}\right)$ (respectively, by $\operatorname{Emb}\left(\Gamma, i_{0}, \mathcal{P}\right)$ and $\left.\mathrm{e}\left(\Gamma, i_{0}, \mathcal{P}\right)\right)$. If $\Omega_{0}=\varnothing$, we omit $i_{0}$ and call the elements of $\operatorname{Emb}_{\text {str }}(\Gamma, \mathcal{P})$ and $\operatorname{Emb}(\Gamma, \mathcal{P})$ strict and nonstrict embeddings of the configuration $\Gamma$ into the plane $\mathcal{P}$. Clearly, $\operatorname{Emb}_{\text {str }}\left(\Gamma, i_{0}, \mathcal{P}\right) \subset \operatorname{Emb}\left(\Gamma, i_{0}, \mathcal{P}\right)$ and

$$
\operatorname{Emb}_{\mathrm{str}}\left(\Gamma, i_{0}, \mathcal{P}\right) \subset \operatorname{Emb}_{\mathrm{str}}(\Gamma, \mathcal{P}), \quad \operatorname{Emb}\left(\Gamma, i_{0}, \mathcal{P}\right) \subset \operatorname{Emb}(\Gamma, \mathcal{P})
$$

for all $i_{0}$. Moreover, if $\operatorname{Emb}_{\mathrm{str}}\left(\Gamma, i_{0}, \mathcal{P}\right) \neq \varnothing$ (respectively, $\left.\operatorname{Emb}\left(\Gamma, i_{0}, \mathcal{P}\right) \neq \varnothing\right)$, then $i_{0}$ is a strict (respectively, nonstrict) embedding of $\Gamma_{\Omega_{0}}$ into $\mathcal{P}$. We observe that the set $\operatorname{Emb}\left(\Gamma, i_{0}, \mathcal{P}\right)$ does not change if we remove some edges of $\Gamma$ belonging to the set $E^{\prime}=\left(E_{\mathfrak{e}^{\prime}} \cup E_{\mathfrak{i}^{\prime}}\right) \backslash \Omega_{0}^{2}$. Below, the elements $\mathfrak{e}$ and $\mathfrak{e}^{\prime}$, as well as $\mathfrak{i}$ and $\mathfrak{i}^{\prime}$, of the set $\mathfrak{T}_{2}$ (see the notation) will be called opposite to each other.

Theorem 4.3. In the above notation, if $i_{0} \in \operatorname{Emb}_{\mathrm{str}}\left(\Gamma_{\Omega_{0}}, \mathcal{P}\right)$, then

$$
\mathrm{e}_{\mathrm{str}}\left(\Gamma, i_{0}, \mathcal{P}\right)=\sum_{S \subset E^{\prime}}(-1)^{|S|} \mathrm{e}\left(\Gamma_{S}, i_{0}, \mathcal{P}\right)
$$

where $\Gamma_{S}$ is obtained from $\Gamma$ by replacing the type of any edge from $S$ with its opposite.

Proof. The definitions of strict and nonstrict extensions imply that

$$
\operatorname{Emb}\left(\Gamma, i_{0}, \mathcal{P}\right) \backslash \operatorname{Emb}_{\text {str }}\left(\Gamma, i_{0}, \mathcal{P}\right)=\bigcup_{s \in E^{\prime}} \operatorname{Emb}\left(\Gamma_{\{s\}}, i_{0}, \mathcal{P}\right)
$$

Since $\Gamma_{\varnothing}=\Gamma$ and $\operatorname{Emb}\left(\Gamma_{S}, i_{0}, \mathcal{P}\right)=\bigcap_{s \in S} \operatorname{Emb}\left(\Gamma_{\{s\}}, i_{0}, \mathcal{P}\right)$ for all nonempty sets $S \subset E^{\prime}$, the required statement follows by the inclusion-exclusion principle. 
Let $\Gamma$ be a configuration on $\Omega$. The connectivity components of the graph $\left(\Omega, E_{\mathfrak{e}}\right)$ induce a partition of $\Omega$. Let $\bar{\Omega}$ denote the set of classes of this partition. Clearly, all elements of a class $A \in \bar{\Omega}$ have the same type; we denote it by $t_{1}(A)$. For a subset $\Omega_{0}$ of $\Omega$, we set

$$
\bar{\Omega}_{0}=\bar{\Omega}_{0, \Gamma}=\left\{A \in \bar{\Omega}: A \cap \Omega_{0} \neq \varnothing\right\} .
$$

The class containing an element (vertex) $\xi \in \Omega$ is denoted by $\bar{\xi}$.

Two elements $\xi, \eta \in \Omega$ (respectively, classes $A, B \in \bar{\Omega}$ ) are said to be incident if $(\xi, \eta) \in E_{\mathfrak{i}}$ (respectively, $\left.(A \times B) \cap E_{\mathfrak{i}} \neq \varnothing\right)$. For $\xi \in \Omega$ and $\Omega_{0} \subset \Omega$ (respectively, for $A \in \bar{\Omega}$ and $\left.\bar{\Omega}_{0} \subset \bar{\Omega}\right)$, we let $N\left(\xi, \Omega_{0}\right)$ (respectively, $N\left(A, \bar{\Omega}_{0}\right)$ ) be the set of all elements of $\Omega_{0}$ (respectively, classes from $\bar{\Omega}_{0}$ ) incident to $\xi$ (respectively, to $A$ ); the cardinality of this set is denoted by $d\left(\xi, \Omega_{0}\right)$ (respectively, by $d\left(A, \bar{\Omega}_{0}\right)$ ). If $\Omega_{0}=\Omega$ (respectively, $\left.\bar{\Omega}_{0}=\bar{\Omega}\right)$, we omit $\Omega_{0}$ (respectively, $\left.\bar{\Omega}_{0}\right)$.

Let $\Gamma$ be a configuration on $\Omega$, and let $\Omega_{0} \subset \Omega, \Gamma_{0}=\Gamma_{\Omega_{0}}$. Then the natural injection from $\Omega_{0}$ to $\Omega$ induces a mapping $f: \bar{\Omega}_{0, \Gamma_{0}} \rightarrow \bar{\Omega}_{\Gamma}$ that preserves the incidence of classes. We say that $\Omega_{0}$ is a $\Gamma$-subset of $\Omega$ if $f$ is injective and two classes $A_{0}, B_{0}$ of $\Gamma_{0}$ are incident whenever the classes $f\left(A_{0}\right)$ and $f\left(B_{0}\right)$ of $\Gamma$ are incident. The configuration $\Gamma$ is said to be final with respect to $\Omega_{0}$ if $\bar{\Omega}_{\Gamma}=\bar{\Omega}_{0, \Gamma}$ and the configuration $\Gamma_{0}$ is complete.

Lemma 4.4. Let $\Gamma=(\Omega, E, t)$ be a final configuration with respect to a set $\Omega_{0} \subset \Omega$, and let $i_{0} \in \operatorname{Emb}_{\text {str }}\left(\Gamma_{0}, \mathcal{P}\right)$, where $\Gamma_{0}=\Gamma_{\Omega_{0}}$ and $\mathcal{P}$ is a projective plane of order $q$. Then $\mathrm{e}\left(\Gamma, i_{0}, \mathcal{P}\right)$ is equal to 0 or 1 , and the latter possibility occurs if and only if $\Omega_{0}$ is a $\Gamma$-subset of $\Omega$.

Proof. Clearly, any nonstrict $\Gamma$-extension of $i_{0}$ maps all elements of any class of $\Gamma$ to one and the same element of $\mathcal{P}$. Thus, the first statement follows from the finality of the configuration $\Gamma$. To prove the second statement, first we suppose that $i$ is a nonstrict $\Gamma$-extension of $i_{0}$. Let $A_{0}, B_{0}$ be classes of $\Gamma_{0}$, and let $A=f\left(A_{0}\right), B=f\left(B_{0}\right)$. If $A$ and $B$ are equal (respectively, incident), then so are the elements $i(A)=i_{0}\left(A_{0}\right)$ and $i(B)=i_{0}\left(B_{0}\right)$ of $\mathcal{P}$. Since the configuration $\Gamma_{0}$ is complete, this implies that the classes $A_{0}$ and $B_{0}$ are equal (respectively, incident). Conversely, let $\Omega_{0}$ be a $\Gamma$-subset of $\Omega$. Since $\Gamma$ is final, the set $A_{0}=A \cap \Omega_{0}$ is nonempty for all $A \in \bar{\Omega}_{\Gamma}$. The injectivity of $f$ shows that $A_{0}$ is a class of $\Gamma_{0}$. Define a mapping $i: \Omega \rightarrow V$, where $V$ is the set of elements of $\mathcal{P}$, by setting $i(\alpha)=i_{0}\left(A_{0}\right)$, where $A_{0}$ is as above with $A$ being the class of $\Gamma$ containing $\alpha$. Clearly, $\left.i\right|_{\Omega_{0}}=i_{0}$. Next, if $(\alpha, \beta) \in E_{\mathfrak{e}}$ (respectively, $(\alpha, \beta) \in E_{\mathfrak{i}}$ ), then the classes $A$ and $B$ are equal (respectively, incident), where $A$ and $B$ are the classes of $\Gamma$ containing $\alpha$ and $\beta$, respectively. Since $\Omega_{0}$ is a $\Gamma$-subset of $\Omega$, this implies that the classes $A_{0}$ and $B_{0}$ are equal (respectively, incident). Therefore, the elements $i(\alpha)=i_{0}\left(A_{0}\right)$ and $i(\beta)=i_{0}\left(B_{0}\right)$ of $\mathcal{P}$ are equal (respectively, incident). Thus, $i \in \operatorname{Emb}\left(\Gamma, i_{0}, \mathcal{P}\right)$.

An important example of a configuration is given by any $\Xi$-configuration and, in particular, by the configuration $\Gamma=\operatorname{Der}(x, \mathcal{P})$, where $x \in V^{4}$ is a quadruple of elements of a projective plane $\mathcal{P}$ (see $\$ 1$ ). We observe that $\Gamma$ is a complete configuration on the set

$$
\Omega_{x}=\Xi \cup\left\{\xi_{k} \xi_{l} \in \Xi^{(2)}: x_{k} \neq x_{l}, t_{1, \mathcal{P}}\left(x_{k}\right)=t_{1, \mathcal{P}}\left(x_{l}\right)\right\},
$$

whence it follows that the mapping

$$
i: \Omega_{x} \rightarrow V, \quad \xi_{j} \mapsto x_{j}, \xi_{k} \xi_{l} \mapsto x_{k} x_{l}
$$

is well defined, where $x_{k} x_{l}$ is the (only) element of $\mathcal{P}$ incident to both $x_{k}$ and $x_{l}$. Set $t_{x}=\left(i \circ t_{1, \mathcal{P}},(i, i) \circ t_{2, \mathcal{P}}\right)$. Then $\Gamma=\left(\Omega_{x}, \Omega_{x}^{2}, t_{x}\right)$ and $i \in \operatorname{Emb}_{\mathrm{str}}(\Gamma, \mathcal{P})$. We call $i$ the embedding of $\Gamma$ associated with $x$. 


\section{$\S 5$. Counting Embedding numbers}

Let $\Gamma=(\Omega, E, t)$ be a configuration. Given $A, B \in \bar{\Omega}$ with $t_{1}(A)=t_{1}(B)$, we set

$$
\Gamma_{A=B}=\left(\Omega, E^{\prime}, t^{\prime}\right), \quad E^{\prime}=E \cup(A \times B) \cup(B \times A), \quad t^{\prime}=\left(t_{1}, t_{2}^{\prime}\right),
$$

where $t_{2}^{\prime}(\xi, \eta)=t_{2}^{\prime}(\eta, \xi)=\mathfrak{e}$ for all $\xi \in A, \eta \in B$, and $t_{2}^{\prime}(\xi, \eta)=t_{2}(\xi, \eta)$ for the other pairs $(\xi, \eta) \in \Omega^{2}$. Let $A, B, C, D$ be pairwise distinct elements of $\bar{\Omega}$. Below we say that $Q=A B C D$ is a quadrangle in $\Gamma$ if each element of the set $\{A, C\}$ is incident to each element of the set $\{B, D\}$.

Lemma 5.1. Let $\Gamma$ be a configuration on $\Omega$, let $\Omega_{0} \subset \Omega$, and let $i_{0} \in \operatorname{Emb}_{\mathrm{str}}\left(\Gamma_{0}, \mathcal{P}\right)$, where $\Gamma_{0}=\Gamma_{\Omega_{0}}$ and $\mathcal{P}$ is a projective plane of order $q$. Suppose that the configuration $\Gamma_{0}$ is complete. Then

1) given $A \in \bar{\Omega} \backslash \bar{\Omega}_{0}$ such that $d(A) \leq 2$, we have

$$
\mathrm{e}\left(\Gamma, i_{0}, \mathcal{P}\right)= \begin{cases}\left(q^{2}+q+1\right) \mathrm{e}\left(\Gamma_{1}, i_{0}, \mathcal{P}\right) & \text { if } d(A)=0, \\ (q+1) \mathrm{e}\left(\Gamma_{1}, i_{0}, \mathcal{P}\right) & \text { if } d(A)=1, \\ \mathrm{e}\left(\Gamma_{1}, i_{0}, \mathcal{P}\right) & \text { if } d(A)=2 \text { and } N\left(A, \bar{\Omega}_{0}\right) \subset \bar{\Omega}_{0}, \\ \mathrm{e}\left(\Gamma_{1}, i_{0}, \mathcal{P}\right)+q \mathrm{e}\left(\Gamma_{2}, i_{0}, \mathcal{P}\right) & \text { otherwise, }\end{cases}
$$

where $\Gamma_{1}=\Gamma_{\Omega \backslash A}, \Gamma_{2}=\left(\Gamma_{1}\right)_{B=C}$ with $N(A)=\{B, C\}$;

2) given a quadrangle $Q=A B C D$ in $\Gamma$, not all vertices of which are in $\bar{\Omega}_{0}$, we have

$$
\begin{aligned}
& \mathrm{e}\left(\Gamma, i_{0}, \mathcal{P}\right)= \begin{cases}\mathrm{e}\left(\Gamma_{1}, i_{0}, \mathcal{P}\right) & \text { if }\{B, D\} \subset \bar{\Omega}_{0}, \\
\mathrm{e}\left(\Gamma_{2}, i_{0}, \mathcal{P}\right) & \text { if }\{A, C\} \subset \bar{\Omega}_{0}, \\
\mathrm{e}\left(\Gamma_{1}, i_{0}, \mathcal{P}\right)+\mathrm{e}\left(\Gamma_{2}, i_{0}, \mathcal{P}\right)-\mathrm{e}\left(\Gamma_{3}, i_{0}, \mathcal{P}\right) & \text { otherwise, }\end{cases} \\
& \text { where } \Gamma_{1}=\Gamma_{A=C}, \Gamma_{2}=\Gamma_{B=D}, \Gamma_{3}=\left(\Gamma_{1}\right)_{B=D} .
\end{aligned}
$$

Proof. We observe that if $p_{1}, p_{2}$ (respectively, $l_{1}, l_{2}$ ) are points (respectively, lines) of the projective plane $\mathcal{P}$ such that $p_{i}$ is incident to $l_{j}$ for all $i, j$, then either $p_{1}=p_{2}$ or $l_{1}=l_{2}$. So, by the definition of nonstrict embedding, we have

$$
\begin{aligned}
\operatorname{Emb}\left(\Gamma, i_{0}, \mathcal{P}\right) & =\operatorname{Emb}\left(\Gamma_{1}, i_{0}, \mathcal{P}\right) \cup \operatorname{Emb}\left(\Gamma_{2}, i_{0}, \mathcal{P}\right), \\
\operatorname{Emb}\left(\Gamma_{3}, i_{0}, \mathcal{P}\right) & =\operatorname{Emb}\left(\Gamma_{1}, i_{0}, \mathcal{P}\right) \cap \operatorname{Emb}\left(\Gamma_{2}, i_{0}, \mathcal{P}\right)
\end{aligned}
$$

Thus the third identity in statement 2 ) is obtained by the inclusion-exclusion principle. If $B, D \in \bar{\Omega}_{0}$, then the completeness of $\Gamma_{0}$ implies that $i(B) \neq i(D)$ for all $i \in \operatorname{Emb}\left(\Gamma, i_{0}, \mathcal{P}\right)$. So $i(A)=i(C)$, whence $\mathrm{e}\left(\Gamma, i_{0}, \mathcal{P}\right)=\mathrm{e}\left(\Gamma_{1}, i_{0}, \mathcal{P}\right)$, which proves the first identity in statement 2$)$. The second line in statement 2 ) is proved similarly.

To prove statement 1 ), we observe that if $d(A)=0$ (respectively, $d(A)=1$ ), then each mapping $i_{1} \in \operatorname{Emb}\left(\Gamma_{1}, i_{0}, \mathcal{P}\right)$ can be extended to $i \in \operatorname{Emb}\left(\Gamma, i_{0}, \mathcal{P}\right)$ in $q^{2}+q+1$ (respectively, $q+1$ ) ways by sending all elements of $A$ to an arbitrary element of $\mathcal{P}$ of type $t_{1}(A)$ (respectively, to an arbitrary element of $\mathcal{P}$ incident to the $i_{1}$-image of the neighbor of $A)$. Thus, $\mathrm{e}\left(\Gamma, i_{0}, \mathcal{P}\right)=\left(q^{2}+q+1\right) \mathrm{e}\left(\Gamma_{1}, i_{0}, \mathcal{P}\right)$ if $d(A)=0$, and $\mathrm{e}\left(\Gamma, i_{0}, \mathcal{P}\right)=$ $(q+1) \mathrm{e}\left(\Gamma_{1}, i_{0}, \mathcal{P}\right)$ if $d(A)=1$. This proves the first two identities. The third identity follows from the completeness of the configuration $\Gamma_{0}$.

Suppose $d(A)=2$ and $\{B, C\} \not \subset \bar{\Omega}_{0}$. For $j=1,2$, denote by $\operatorname{Emb}_{j}\left(\Gamma, i_{0}, \mathcal{P}\right)(\operatorname{respec}-$ tively, $\left.\operatorname{Emb}_{j}\left(\Gamma_{1}, i_{0}, \mathcal{P}\right)\right)$ the set of all $i \in \operatorname{Emb}\left(\Gamma, i_{0}, \mathcal{P}\right)$ (respectively, $i \in \operatorname{Emb}\left(\Gamma_{1}, i_{0}, \mathcal{P}\right)$ ) such that $i(B) \neq i(C)$ for $j=1$, and $i(B)=i(C)$ for $j=2$. Then, obviously,

$$
\begin{aligned}
\operatorname{Emb}\left(\Gamma, i_{0}, \mathcal{P}\right) & =\operatorname{Emb}_{1}\left(\Gamma, i_{0}, \mathcal{P}\right) \cup \operatorname{Emb}_{2}\left(\Gamma, i_{0}, \mathcal{P}\right), \\
\operatorname{Emb}\left(\Gamma_{1}, i_{0}, \mathcal{P}\right) & =\operatorname{Emb}_{1}\left(\Gamma_{1}, i_{0}, \mathcal{P}\right) \cup \operatorname{Emb}_{2}\left(\Gamma_{1}, i_{0}, \mathcal{P}\right),
\end{aligned}
$$


and the unions are disjoint. Set $\mathrm{e}_{j}\left(\Gamma, i_{0}, \mathcal{P}\right)=\left|\operatorname{Emb}_{j}\left(\Gamma, i_{0}, \mathcal{P}\right)\right|$ and $\mathrm{e}_{j}\left(\Gamma_{1}, i_{0}, \mathcal{P}\right)=$ $\left|\operatorname{Emb}_{j}\left(\Gamma_{1}, i_{0}, \mathcal{P}\right)\right|$. Since two distinct elements of the same type in a projective plane are incident to a unique element of the other type, each $i_{1} \in \operatorname{Emb}_{1}\left(\Gamma_{1}, i_{0}, \mathcal{P}\right)$ extends uniquely to $i \in \operatorname{Emb}_{1}\left(\Gamma, i_{0}, \mathcal{P}\right)$. So,

$$
\mathrm{e}_{1}\left(\Gamma, i_{0}, \mathcal{P}\right)=\mathrm{e}_{1}\left(\Gamma_{1}, i_{0}, \mathcal{P}\right) .
$$

On the other hand, each $i_{1} \in \operatorname{Emb}_{2}\left(\Gamma_{1}, i_{0}, \mathcal{P}\right)$ extends to $i \in \operatorname{Emb}_{2}\left(\Gamma, i_{0}, \mathcal{P}\right)$ in $q+1$ ways by sending all elements of $A$ to an arbitrary element of $\mathcal{P}$ incident to $i_{1}(B)=i_{1}(C)$. Since, obviously, $\mathrm{e}_{2}\left(\Gamma_{1}, i_{0}, \mathcal{P}\right)=\mathrm{e}\left(\Gamma_{2}, i_{0}, \mathcal{P}\right)$, this implies that

$$
\mathrm{e}_{2}\left(\Gamma, i_{0}, \mathcal{P}\right)=(q+1) \mathrm{e}_{2}\left(\Gamma_{1}, i_{0}, \mathcal{P}\right)=\mathrm{e}_{2}\left(\Gamma_{1}, i_{0}, \mathcal{P}\right)+q \mathrm{e}\left(\Gamma_{2}, i_{0}, \mathcal{P}\right) .
$$

By (14) and (15), we have $\mathrm{e}\left(\Gamma, i_{0}, \mathcal{P}\right)=\mathrm{e}\left(\Gamma_{1}, i_{0}, \mathcal{P}\right)+q \mathrm{e}\left(\Gamma_{2}, i_{0}, \mathcal{P}\right)$, which proves the third identity in statement 1 ).

Throughout the rest of the section we fix a set $\Omega_{0}$ and denote by $\Gamma$ the class of all configurations on sets containing $\Omega_{0}$. Let $\Gamma \in \boldsymbol{\Gamma}$. Given a class $A$ of $\Gamma$ not in $\bar{\Omega}_{0}$ with $d(A) \leq 2$, we set

$$
N(\Gamma, A)= \begin{cases}\left\{\Gamma_{1}\right\} & \text { if } d(A) \leq 1 \text { or } N(A) \subset \bar{\Omega}_{0}, \\ \left\{\Gamma_{1}, \Gamma_{2}\right\} & \text { otherwise, }\end{cases}
$$

where $\Gamma_{1}=\Gamma_{\Omega \backslash A}$ and $\Gamma_{2}=\Gamma_{B=C}$ with $B$ and $C$ being the neighbors of $A$ (cf. statement 1) of Lemma 5.11). Similarly, given a quadrangle $Q=A B C D$ in $\Gamma$, not all vertices of which are in $\bar{\Omega}_{0}$, we set

$$
N(\Gamma, Q)= \begin{cases}\left\{\Gamma_{1}\right\} & \text { if }\{B, D\} \subset \bar{\Omega}_{0}, \\ \left\{\Gamma_{2}\right\} & \text { if }\{A, C\} \subset \bar{\Omega}_{0}, \\ \left\{\Gamma_{1}, \Gamma_{2}, \Gamma_{3}\right\} & \text { otherwise }\end{cases}
$$

where $\Gamma_{1}=\Gamma_{A=C}, \Gamma_{2}=\Gamma_{B=D}$ and $\Gamma_{3}=\left(\Gamma_{1}\right)_{B=D}$ (cf. statement 2) of Lemma 5.1). Clearly, $N(\Gamma, A), N(\Gamma, Q)$ are subsets of $\boldsymbol{\Gamma}$.

Definition 5.2. By a $d$-deduction in $\Gamma$ we mean a rooted tree of depth $d$ with vertices in $\boldsymbol{\Gamma}$ such that the set of sons of any nonleaf $\Gamma^{\prime}$ is equal either to $N\left(\Gamma^{\prime}, A\right)$ for some class $A$ of $\Gamma^{\prime}$ not in $\bar{\Omega}_{0, \Gamma^{\prime}}$ with $d(A) \leq 2$, or to $N\left(\Gamma^{\prime}, Q\right)$ for some quadrangle $Q$ in $\Gamma^{\prime}$ not all vertices of which are in $\bar{\Omega}_{0, \Gamma^{\prime}}$. If $\Gamma$ is the root of this tree and a set $\Gamma^{\prime} \subset \boldsymbol{\Gamma}$ contains all of its leaves, we say that it is a $d$-deduction of $\Gamma$ from $\Gamma^{\prime}$. In this case for any $d^{\prime} \geq d$ we also say that $\Gamma$ is $d^{\prime}$-deducible from $\Gamma^{\prime}$.

This definition shows that $\Gamma$ is 0 -deducible from $\Gamma^{\prime}$ if and only if $\Gamma \in \Gamma^{\prime}$. Also, if $\Gamma$ is $d_{1}$-deducible from a set $\boldsymbol{\Gamma}_{1}$, each element of which is $d_{2}$-deducible from $\boldsymbol{\Gamma}_{2}$, then $\Gamma$ is $\left(d_{1}+d_{2}\right)$-deducible from $\boldsymbol{\Gamma}_{2}$. Applying Lemmas 5.1 and 4.4 and Theorem 4.3 , we immediately obtain the following statement.

Theorem 5.3. Suppose $\Gamma \in \Gamma$ and $i_{0} \in \operatorname{Emb}_{\text {str }}\left(\Gamma_{\Omega_{0}}, \mathcal{P}\right)$, where $\mathcal{P}$ is a projective plane of order $q$. Suppose also that for some $d \geq 0$ the configuration $\Gamma$ is d-deducible from the set of all final configurations belonging to $\boldsymbol{\Gamma}$. Then

$$
\mathrm{e}_{\text {str }}\left(\Gamma, i_{0}, \mathcal{P}\right)=f_{\Gamma}(q), \quad \mathrm{e}\left(\Gamma, i_{0}, \mathcal{P}\right)=g_{\Gamma}(q),
$$

where $f_{\Gamma}(x)$ and $g_{\Gamma}(x)$ are polynomials with integral coefficients independent of $\mathcal{P}$ and $i_{0}$. 


\section{§6. Proof of Theorem 1.1}

Let $\Gamma=(\Omega, E, t)$, where $t=\left(t_{1}, t_{2}\right)$, be a $\Xi$-configuration (see $\left.₫ 4\right)$. It is easily seen that $R(\Gamma, \mathcal{P})^{T}=R\left(\Gamma^{T}, \mathcal{P}\right)$, where $\Gamma^{T}=\left(\Omega, E, t^{\prime}\right)$ with $t^{\prime}=\left(f_{1} \circ t_{1}, f_{2} \circ t_{2}\right)$ and $f_{1} \in \operatorname{Sym}(\Omega)$, $f_{2} \in \operatorname{Sym}(E)$ are the permutations induced by the involution $\left(\xi_{1}, \xi_{3}\right)\left(\xi_{2}, \xi_{4}\right) \in \operatorname{Sym}(\Xi)$. Also,

$$
\Delta\left(V^{2}\right)=\bigcup_{\Gamma} R(\Gamma, \mathcal{P}),
$$

where $\Gamma$ runs over all $\Xi$-configurations such that $t_{2}\left(\xi_{1}, \xi_{3}\right)=t_{2}\left(\xi_{2}, \xi_{4}\right)=\mathfrak{e}$. Thus, the partition $\Pi$ is closed with respect to transposition, and the set $\Delta\left(V^{2}\right)$ is a union of classes of $\Pi$. To complete the proof of statement 1 ), it only suffices to verify that for any $R, S, T \in \Pi$, the number $c_{R, S}(u, w)$ defined by (5) does not depend on the choice of $(u, w) \in T$. We need some notation.

Let $\Sigma$ be the set of six distinct commuting variables $\xi_{1}, \xi_{2}, \xi_{3}, \xi_{4}$ and $\sigma, \tau$. Thus, $\Sigma=\Xi \cup\{\sigma, \tau\}$, where $\Xi$ is the set defined in $\S 1$ Put $\boldsymbol{\Omega}=\Sigma \cup \Sigma^{(2)}$, where $\Sigma^{(2)}$ is the symmetric square of $\Sigma$. Obviously, $|\boldsymbol{\Omega}|=21$ and $\boldsymbol{\Omega}$ contains the set $\boldsymbol{\Omega}_{0}=\Xi \cup \Xi^{(2)}$. For $k=0,1,2$, we introduce an injection $f_{k}: \boldsymbol{\Omega}_{0} \rightarrow \boldsymbol{\Omega}$ such that

$$
\left(f_{k}\left(\xi_{1}\right), f_{k}\left(\xi_{2}\right), f_{k}\left(\xi_{3}\right), f_{k}\left(\xi_{4}\right)\right)= \begin{cases}\left(\xi_{1}, \xi_{2}, \xi_{3}, \xi_{4}\right) & \text { if } k=0, \\ \left(\xi_{1}, \xi_{2}, \sigma, \tau\right) & \text { if } k=1, \\ \left(\sigma, \tau, \xi_{3}, \xi_{4}\right) & \text { if } k=2,\end{cases}
$$

and $f_{k}\left(\xi_{i} \xi_{j}\right)=f_{k}\left(\xi_{i}\right) f_{k}\left(\xi_{j}\right)$ for all $i \neq j$. Set $\boldsymbol{\Omega}_{k}=f_{k}\left(\boldsymbol{\Omega}_{0}\right)(k=1,2)$.

Let $\Gamma_{k}$ be a $\Xi$-configuration, $k=0,1,2$. Denote by $\Gamma_{k}^{\prime}$ the configuration obtained from $\Gamma_{k}$ by translation of the structure along $f_{k}$. Obviously, $\Gamma_{0}^{\prime}=\Gamma_{0}$. Suppose that the configurations $\Gamma_{0}, \Gamma_{1}, \Gamma_{2}$ are consistent, i.e.,

$$
\left(\Gamma_{k}^{\prime}\right)_{\Omega_{k} \cap \Omega_{l}}=\left(\Gamma_{l}^{\prime}\right)_{\Omega_{k} \cap \Omega_{l}}, \quad k, l=0,1,2,
$$

where $\Omega_{k}$ is the underlying set of $\Gamma_{k}^{\prime}$. Then there exists a uniquely determined configuration $\Gamma$ on the set $\Omega=\bigcup_{k=0}^{2} \Omega_{k}$ such that $\Gamma_{\Omega_{k}}=\Gamma_{k}^{\prime}$ for all $k$. We denote it by $\left[\Gamma_{0}, \Gamma_{1}, \Gamma_{2}\right]$ and call it the initial configuration associated with $\Gamma_{0}, \Gamma_{1}, \Gamma_{2}$. The following statement is straightforward from the definitions.

Lemma 6.1. Let $\mathcal{P}$ be a projective plane, let $V$ be the set of its elements, and let $\Gamma_{0}, \Gamma_{1}, \Gamma_{2}$ be $\Xi$-configurations with $\Gamma_{0}=\operatorname{Der}(x, \mathcal{P})$, where $x \in V^{4}$. Suppose that the configurations $\Gamma_{0}, \Gamma_{1}, \Gamma_{2}$ are consistent and $\Gamma=\left[\Gamma_{0}, \Gamma_{1}, \Gamma_{2}\right]$. Then for all $R=R\left(\Gamma_{1}, \mathcal{P}\right)$, $S=R\left(\Gamma_{2}, \mathcal{P}\right)$ and $u=\left(x_{1}, x_{2}\right), w=\left(x_{3}, x_{4}\right)$ we have

$$
c_{R, S}(u, w)=\mathrm{e}_{\mathrm{str}}\left(\Gamma, i_{0}, \mathcal{P}\right),
$$

where $i_{0} \in \mathrm{Emb}_{\text {str }}\left(\Gamma_{0}, \mathcal{P}\right)$ is the embedding associated with $x$.

We complete the proof of statement 1$)$. Take $R, S, T \in \Pi$ and set $\Gamma_{0}=\operatorname{Der}(T), \Gamma_{1}=$ $\operatorname{Der}(R), \Gamma_{2}=\operatorname{Der}(S)$. If $\Gamma_{0}, \Gamma_{1}$, and $\Gamma_{2}$ are not consistent, then, obviously, $c_{R, S}(u, w)=$ 0 for all $(u, w) \in T$. Suppose that they are consistent and take $(u, w) \in T$. Then $\Gamma_{0}=\operatorname{Der}(x, \mathcal{P})$, where $x=(u, w)$. By Lemma 6.1 we have $c_{R, S}(u, w)=\mathrm{e}_{\text {str }}\left(\Gamma, i_{0}, \mathcal{P}\right)$ with $\Gamma$ and $i_{0}$ as in the lemma. By Theorem 6.2 below, the latter number does not depend on the choice of $(u, w)$, which proves statement 1$)$.

Theorem 6.2. Let $\Gamma$ be an initial configuration, and let $i_{0} \in \operatorname{Emb}_{\mathrm{str}}\left(\Gamma_{0}, \mathcal{P}\right)$, where $\mathcal{P}$ is a projective plane of order $q$. Then

$$
\mathrm{e}_{\mathrm{str}}\left(\Gamma, i_{0}, \mathcal{P}\right)=f_{\Gamma}(q)
$$

where $f_{\Gamma}(x)$ is a polynomial with integral coefficients not depending on $\mathcal{P}$ and $i_{0}$. 
Proof. This follows from Theorems 7.3 and 7.4 to be proved in the next section, in combination with Theorem 5.3 .

Statement 3) of Theorem 1.1 is obtained from Theorem 6.2 as a byproduct. To prove statement 2), let $\Gamma^{\prime} \in \operatorname{Der}(\mathcal{P})$. Then there exists $x \in V^{4}$ such that $\Gamma^{\prime}=\operatorname{Der}(x, \mathcal{P})$. Set $\Gamma_{0}=\operatorname{Der}(y, \mathcal{P})$, where $y=\left(x_{1}, x_{2}, x_{1}, x_{2}\right), \Gamma_{1}=\Gamma^{\prime}$, and $\Gamma_{2}=\left(\Gamma^{\prime}\right)^{T}$. Since, obviously, these configurations are consistent, we can form the initial configuration $\Gamma=\left[\Gamma_{0}, \Gamma_{1}, \Gamma_{2}\right]$. Then

$$
\begin{aligned}
R\left(\Gamma_{0}, \mathcal{P}\right)=\left\{\left(z_{1}, z_{2}, z_{1}, z_{2}\right) \in V^{4}:\right. & \\
& \left.t_{1}\left(z_{1}\right)=t_{1}\left(x_{1}\right), t_{1}\left(z_{2}\right)=t_{1}\left(x_{2}\right), t_{2}\left(z_{1}, z_{2}\right)=t_{2}\left(x_{1}, x_{2}\right)\right\} .
\end{aligned}
$$

Take another projective plane $\mathcal{P}^{\prime}$ of order $q^{\prime}$ and elements $x_{1}^{\prime}, x_{2}^{\prime} \in V^{\prime}$ such that $t_{1}\left(x_{1}^{\prime}\right)=$ $t_{1}\left(x_{1}\right), t_{1}\left(x_{2}^{\prime}\right)=t_{1}\left(x_{2}\right), t_{2}\left(x_{1}^{\prime}, x_{2}^{\prime}\right)=t_{2}\left(x_{1}, x_{2}\right)$. From (16) it follows that $\operatorname{Der}(y, \mathcal{P})=$ $\operatorname{Der}\left(y^{\prime}, \mathcal{P}^{\prime}\right)=\Gamma_{0}$, where $y^{\prime}=\left(x_{1}^{\prime}, x_{2}^{\prime}, x_{1}^{\prime}, x_{2}^{\prime}\right)$. We denote by $i_{0} \in \operatorname{Emb}_{\text {str }}\left(\Gamma_{0}, \mathcal{P}\right)$ and $i_{0}^{\prime} \in \operatorname{Emb}_{\text {str }}\left(\Gamma_{0}, \mathcal{P}^{\prime}\right)$ the embeddings associated with the quadruples $y$ and $y^{\prime}$, respectively. Then, by Theorem 6.2, we have

$$
\mathrm{e}_{\mathrm{str}}\left(\Gamma, i_{0}, \mathcal{P}\right)=f_{\Gamma}(q), \quad \mathrm{e}_{\mathrm{str}}\left(\Gamma, i_{0}^{\prime}, \mathcal{P}^{\prime}\right)=f_{\Gamma}\left(q^{\prime}\right),
$$

where $q^{\prime}$ is the order of $\mathcal{P}^{\prime}$. Since, obviously, $\mathrm{e}_{\text {str }}\left(\Gamma, i_{0}, \mathcal{P}\right) \neq 0$, the polynomial $f_{\Gamma}$ is nonzero. Therefore, $\mathrm{e}_{\mathrm{str}}\left(\Gamma_{1}, \mathcal{P}^{\prime}\right) \neq 0$ whenever $q^{\prime}=q$ or $q^{\prime}$ is larger than the maximum absolute value of a root of $f_{\Gamma}$. In both cases we have $R\left(\Gamma^{\prime}, \mathcal{P}^{\prime}\right)=R\left(\Gamma_{1}, \mathcal{P}^{\prime}\right) \neq \varnothing$, whence it follows that $\Gamma^{\prime} \in \operatorname{Der}\left(\mathcal{P}^{\prime}\right)$. This proves the first part of statement 2), and also the second part, because the number of all $\Xi$-configurations is finite.

\section{$\S 7$. Admissible configurations}

7.1. Let $\Gamma$ be an initial configuration on $\Omega$ (see $\sqrt{6}$ ). The definition implies that $\Omega \subset \Omega$ and

$$
\Gamma_{\Omega_{0}} \text { is a } \Xi \text {-configuration. }
$$

Also, $\Gamma$ satisfies the following condition for $\xi, \eta, \zeta \in \Sigma$ :

$$
\xi, \xi \eta \in \Omega \Rightarrow t_{2}(\xi, \xi \eta)=\mathfrak{i}, \quad \text { and } \xi \eta, \xi \zeta \in \Omega \Rightarrow t_{1}(\xi \eta)=t_{1}(\xi \zeta)
$$

(this is an easy consequence of the fact that the same is true for any $\Xi$-configuration on $\Omega$ and $\xi, \eta, \zeta \in \Xi$ ). Moreover, conditions (17) and (18) are preserved when we pass from a configuration $\Gamma$ to the configuration $\Gamma_{\Omega \backslash A}$ with $A \in \bar{\Omega} \backslash \bar{\Omega}_{0}$, as well as to the configuration $\Gamma_{A=B}$ with $A, B \in \bar{\Omega}$ such that $\{A, B\} \not \subset \bar{\Omega}_{0}$.

From now on, by a configuration we mean any configuration $\Gamma$ on $\Omega \subset \Omega$ satisfying conditions (17) and (18) (we will refer to (18) as the T-argument or the type comparison argument). We set

$$
\Omega_{1}^{*}=\Omega \cap\left\{\sigma \xi_{1}, \sigma \xi_{2}, \tau \xi_{1}, \tau \xi_{2}\right\}, \quad \Omega_{2}^{*}=\Omega \cap\left\{\sigma \xi_{3}, \sigma \xi_{4}, \tau \xi_{3}, \tau \xi_{4}\right\} .
$$

Clearly, $\Omega_{i}^{*}=\Omega_{i} \backslash\left(\Omega_{0} \cap \Omega_{3-i}\right), i=1,2$.

Definition 7.1. A configuration $\Gamma$ on $\Omega$ is said to be admissible at a class $A \in \bar{\Omega}$ if either $A \not \subset \Omega_{1}^{*} \cup \Omega_{2}^{*}$ or

$$
A \cap \Omega_{i}^{*} \neq \varnothing \Rightarrow N(A) \subset \bar{\Omega}_{i}, \quad i=1,2 .
$$

The configuration $\Gamma$ is said to be admissible if it is admissible at any of its classes.

The next lemma follows straightforwardly from the definition. 
Lemma 7.2. If a configuration $\Gamma$ on $\Omega$ is admissible at a class $A \in \bar{\Omega}$, then so are the configurations $\Gamma_{\Omega \backslash B}$ and $\Gamma_{C=D}$, where $B, C, D$ are classes of $\Gamma$ other than $A$ and such that $B$ and at least one of $C, D$ belong to $\bar{\Omega} \backslash \bar{\Omega}_{0}, 4$

Theorem 7.3. Any initial configuration is admissible.

Proof. Let $\Gamma$ be an initial configuration associated with the $\Xi$-configurations $\Gamma_{0}, \Gamma_{1}$, and $\Gamma_{2}$. It suffices to verify that $\Gamma$ is admissible at each class $A \in \bar{\Omega}$ such that $A \subset \Omega_{1}^{*} \cup \Omega_{2}^{*}$. Suppose that $A \cap \Omega_{i}^{*} \neq \varnothing$ for some $i=1,2$. If $A \cap \Omega_{3-i}^{*} \neq \varnothing$, then we can find $\alpha_{0}, \ldots, \alpha_{k} \in A$ such that $t_{2}\left(\alpha_{j}, \alpha_{j+1}\right)=\mathfrak{e}$ for all $j=0, \ldots, k-1$, where $\alpha_{0} \in \Omega_{i}^{*}$ and $\alpha_{k} \in \Omega_{3-i}^{*}$. Set $l=\max _{\alpha_{j} \in \Omega_{i}^{*}} j$. Then $l<k$ and $\alpha_{l+1} \notin \Omega_{i}^{*}$. On the other hand, $\alpha_{l} \in \Omega_{i}^{*}$, so that the definition of an initial configuration implies that $\alpha_{l+1} \in \Omega_{i}$, a contradiction. We conclude that $A \subset \Omega_{i}^{*}$, whence it follows that $N(\alpha) \subset \Omega_{i}$ for all $\alpha \in A$. Thus, $N(A) \subset \bar{\Omega}_{i}$.

Let $d>0$. An admissible configuration $\Gamma$ on $\Omega$ is said to be $d$-reducible if there exists a $d^{\prime}$-deduction of it from the admissible configurations in the class of all configurations, the underlying sets of which contain $\Omega_{0}$, where $0<d^{\prime} \leq d$ (see Definition 5.2). In 88 we shall prove the following theorem.

Theorem 7.4. Each admissible configuration other than a final one is 2-reducible. In particular, any admissible configuration is d-deducible from final ones for some $d$.

7.2. In this subsection we find sufficient conditions for an admissible configuration to be 1-reducible or 2-reducible (Theorem 7.7 and Lemma 7.8). For this purpose, the following definition is useful.

Definition 7.5. Let $\Gamma$ be an admissible configuration on $\Omega$. We say that two classes $A_{1}, A_{2} \in \bar{\Omega}$ of the same type are comparable (respectively, quasicomparable) in $\Gamma$ if either $A_{1}, A_{2} \in \bar{\Omega}_{0}$, or the configuration $\Gamma_{A_{1}=A_{2}}$ is admissible (respectively, 1-deducible from admissible ones).

Below we frequently use the following conditions sufficient for comparability and quasicomparability.

Lemma 7.6. Let $\Gamma$ be an admissible configuration on $\Omega$. Suppose that $A_{1}, A_{2} \in \bar{\Omega}$ are classes of the same type and that one of them does not belong to $\bar{\Omega}_{0}$. Then:

1) $A_{1}$ and $A_{2}$ are comparable whenever either $A_{i} \not \subset \Omega_{1}^{*} \cup \Omega_{2}^{*}$ for some $i$, or $N\left(A_{1}\right)=$ $N\left(A_{2}\right)$,

2) $A_{1}$ and $A_{2}$ are quasicomparable whenever $A_{1}, A_{2} \notin \bar{\Omega}_{0}$ and $\Gamma$ contains a path $P=A_{1} B C D A_{2}$ with $C \in \bar{\Omega}_{0}$ such that either $B, D \in \bar{\Omega}_{0}$, or $N\left(A_{1}\right) \backslash\{B\}=$ $N\left(A_{2}\right) \backslash\{D\}$ and $N(B) \backslash\left\{A_{1}\right\}=N(D) \backslash\left\{A_{2}\right\}$.

Proof. Lemma 7.2 shows that the configuration $\Gamma^{\prime}=\Gamma_{A_{1}=A_{2}}$ is admissible at each class other than $A=A_{1} \cup A_{2}$. Suppose that the hypothesis of statement 1) is fulfilled. If $A_{i} \not \subset \Omega_{1}^{*} \cup \Omega_{2}^{*}$ for some $i$, then, obviously, $\Gamma^{\prime}$ is admissible at $A$ (Definition 7.1), and hence, the classes $A_{1}$ and $A_{2}$ are comparable. Otherwise, the neighborhood of $A$ in $\Gamma^{\prime}$ coincides with $N\left(A_{1}\right)=N\left(A_{2}\right)$. Since $\Gamma$ is admissible at both $A_{1}$ and $A_{2}$, this implies that $\Gamma^{\prime}$ is admissible at $A$ (see (20) $)$. Statement 1) is proved.

Suppose that the hypothesis of statement 2) is fulfilled. Then $Q=A B C D$ is a quadrangle in $\Gamma^{\prime}$. Moreover, since $C \in \bar{\Omega}_{0}$, the configuration $\Gamma_{1}^{\prime}=\Gamma_{A=C}^{\prime}$ is admissible at the class $A \cup C$; hence, it is admissible by Lemma 7.2. If $B, D \in \bar{\Omega}_{0}$, then $\Gamma^{\prime}$ is 1-deducible from $\Gamma_{1}^{\prime}$ and we are done. Otherwise, $\Gamma^{\prime}$ is 1-deducible from $\Gamma_{1}^{\prime}, \Gamma_{2}^{\prime}=\Gamma_{B=D}^{\prime}$, and $\Gamma_{3}^{\prime}=\left(\Gamma_{2}^{\prime}\right)_{A=C}=\left(\Gamma_{1}^{\prime}\right)_{B=D}$. Therefore, by Lemma 7.2 it suffices to verify that

${ }^{4}$ Obviously, $A$ is a class of each of the configurations $\Gamma_{\Omega \backslash B}$ and $\Gamma_{C=D}$. 
the configuration $\Gamma_{2}^{\prime}$ is admissible. However, by the same lemma, this configuration is admissible at each class other than $A$ and $B \cup D$. From the hypothesis it follows that the neighborhoods of $A_{1}$ and $A_{2}$ in $\Gamma^{\prime \prime}=\Gamma_{B=D}$ coincide. Since $\Gamma^{\prime \prime}$ is admissible at both $A_{1}$ and $A_{2}$, this implies that the configuration $\Gamma_{2}^{\prime}=\Gamma_{A_{1}=A_{2}}^{\prime \prime}$ is admissible at the class $A$ (see the preceding paragraph). Similarly, the neighborhoods of $B$ and $D$ in $\Gamma^{\prime}$ coincide and $\Gamma^{\prime}$ is admissible at both $B$ and $D$. Thus, the configuration $\Gamma_{2}^{\prime}$ is admissible at the class $B \cup D$.

Theorem 7.7. Let $\Gamma$ be an admissible configuration on $\Omega$. Then the following two statements hold true:

1) Let $A \in \bar{\Omega} \backslash \bar{\Omega}_{0}$ and $d(A) \leq 2$. Suppose that the neighbors of $A$ are comparable (respectively, quasicomparable) in $\Gamma_{\Omega \backslash A}$ whenever $d(A)=2$. Then $\Gamma$ is 1-reducible (respectively, 2-reducible).

2) Let $Q=A B C D$ be a quadrangle in $\Gamma$, not all vertices of which belong to $\bar{\Omega}_{0}$. Suppose that $A$ and $C$ are comparable in $\Gamma$ (respectively, quasicomparable in $\Gamma$ and also in $\Gamma_{B=D}$ if $B$ or $D$ does not belong to $\left.\bar{\Omega}_{0}\right)$. Then $\Gamma$ is 1-reducible (respectively, 2-reducible) whenever $B$ and $D$ are comparable.

Proof. We prove statement 1). Since $d(A) \leq 2$, the configuration $\Gamma$ is 1-deducible from the set $\left\{\Gamma_{1}\right\}$ if $d(A)<2$, and from the set $\left\{\Gamma_{1}, \Gamma_{2}\right\}$ if $d(A)=2$, where $\Gamma_{1}=\Gamma_{\Omega \backslash A}$, and $\Gamma_{2}=\left(\Gamma_{1}\right)_{B=C}, B$ and $C$ being the neighbors of $A$. By Lemma 7.2 , the configuration $\Gamma_{1}$ is admissible. Suppose that $\Gamma_{2}$ enters the deduction. Then at least one of $B$ and $C$ does not belong to $\bar{\Omega}_{0, \Gamma_{1}}=\bar{\Omega}_{0} \backslash\{A\}$. So, if the classes $B$ and $C$ are comparable (respectively, quasicomparable) in $\Gamma_{1}$, then, by Definition [7.1, the configuration $\Gamma_{2}$ is admissible (respectively, 1-deducible from admissible ones), and hence the configuration $\Gamma$ is 1-reducible (respectively, 2-reducible).

Now we prove statement 2). Since not all vertices of $Q$ belong to $\bar{\Omega}_{0}$, the configuration $\Gamma$ is 1-deducible from the set $\left\{\Gamma_{1}, \Gamma_{2}, \Gamma_{3}\right\}$, where $\Gamma_{1}=\Gamma_{A=C}, \Gamma_{2}=\Gamma_{B=D}$, and $\Gamma_{3}=$ $\left(\Gamma_{1}\right)_{B=D}=\left(\Gamma_{2}\right)_{A=C}$. If $\Gamma_{1}$ enters the deduction, then either $A$ or $C$ does not belong to $\bar{\Omega}_{0}$. So, by Definition 7.5 the configuration $\Gamma_{1}$ is admissible (respectively, 1-deducible from admissible ones) whenever the classes $A$ and $C$ are comparable (respectively, quasicomparable) in $\Gamma$. Suppose that the classes $B$ and $D$ are comparable in $\Gamma$. Then if $\Gamma_{2}$ enters the deduction, it is admissible by the above argument. Suppose $\Gamma_{3}$ enters the deduction. Then so do $\Gamma_{1}$ and $\Gamma_{2}$. Therefore, if $A$ and $C$ are comparable in $\Gamma$, then these configurations are admissible by the above, and hence, by Lemma 7.2 , the configuration $\Gamma_{3}$ is admissible. Finally, let $A$ and $C$ be quasicomparable in $\Gamma$. Since either $B$ or $D$ does not belong to $\bar{\Omega}_{0}$, the hypothesis of statement 2) implies that $A$ and $C$ are quasicomparable in $\Gamma_{2}$, and hence, the configuration $\Gamma_{3}$ is 1-deducible from admissible configurations. Thus, in the comparability (respectively, quasicomparability) case, the configuration $\Gamma$ is 1-deducible (respectively, 2-deducible) from admissible configurations; i.e., it is 1-reducible (respectively, 2-reducible).

The sufficient condition of 1-reducibility (respectively, 2-reducibility) occurring in statements 1) and 2) of Theorem 7.7 will be referred to as the D-argument and the $Q$-argument (respectively, the generalized $D$-argument and the generalized $Q$-argument). Statement 1) of Lemma 7.6 shows that two classes of a configuration $\Gamma$ are comparable whenever one of them belongs to the set

$$
X^{*}=X \backslash \bar{\Omega}_{1} \cap \Omega_{2}, \quad X=\bar{\Omega} \backslash \bar{\Omega}_{0} .
$$

In these cases we shall use the D-argument and the Q-argument without any reference to the above statement (and even without any mention that the classes are comparable). 
Below we shall use the following property of an admissible but not 1-reducible configuration $\Gamma$ on $\Omega$ : given $A \in X$ and $\xi, \eta \in \Omega_{0}$, we have

$$
\bar{\xi}, \bar{\eta} \in N\left(A, \bar{\Omega}_{0}\right), \bar{\xi} \neq \bar{\eta} \Rightarrow\{\xi, \eta\}=\left\{\xi_{i} \xi_{j}, \xi_{k}\right\} \text { or }\{\xi, \eta\}=\left\{\xi_{i} \xi_{j}, \xi_{k} \xi_{l}\right\}
$$

with distinct $i, j, k, l \in\{1,2,3,4\}$. Indeed, if $\underline{\xi}=\xi_{i}, \eta=\xi_{j}$ (respectively, $\xi=\xi_{i} \xi_{j}, \eta=$ $\left.\xi_{i} \xi_{k}\right)$, then, by the Q-argument with $Q=A \bar{\xi}_{i} \overline{\xi_{i} \xi_{j}} \bar{\xi}_{j}$ (respectively, $Q=A \overline{\xi_{i} \xi_{j}} \bar{\xi}_{i} \overline{\xi_{i} \xi_{k}}$ ), the configuration $\Gamma$ is 1-reducible. We observe that $t_{1}(\xi)=t_{1}(\eta)$. So, by the T-argument, the set $\{\xi, \eta\}$ cannot contain both a variable and the product of this variable by another variable. This proves (22).

Lemma 7.8. Let $\Gamma$ be an admissible configuration on $\Omega$. Suppose that at least one of the following conditions is satisfied:

1) $d\left(A, \bar{\Omega}_{0}\right) \geq 3$ for some $A \in X$;

2) $d\left(A, \bar{\Omega}_{0}\right)+d\left(B, \bar{\Omega}_{0}\right) \geq 4$ for some incident classes $A, B \in X$;

3) $d\left(B, \bar{\Omega}_{0}\right)+d\left(C, \bar{\Omega}_{0}\right) \geq 4$ and $d\left(A, \bar{\Omega}_{0}\right) \geq 1$ for some distinct comparable classes $B, C \in X$ such that $A$ is incident to both $B$ and $C$.

Then the configuration $\Gamma$ is 1-reducible.

Proof. Let $\bar{\xi}, \bar{\eta}, \bar{\zeta}$ be pairwise distinct elements of $N\left(A, \bar{\Omega}_{0}\right)$, where $\xi, \eta, \zeta \in \Omega_{0}$. Since all 2-subsets of the set $\{\xi, \eta, \zeta\}$ cannot simultaneously be of the form (22), statement 1) follows. We prove statement 2). By statement 1$)$, we may assume that $d\left(A, \bar{\Omega}_{0}\right)=$ $d\left(B, \bar{\Omega}_{0}\right)=2$. From (22) (applied to $A$ and $B$ ) it follows that $\bar{\xi}_{i} \xi_{j} \in N\left(A, \bar{\Omega}_{0}\right)$ and $\overline{\xi_{k} \xi_{l}} \in$ $N\left(B, \bar{\Omega}_{0}\right)$ for some $i, j, k, l$. Then the T-argument shows that $\{i, j, k, l\}=\{1,2,3,4\}$ and that the neighbor of $A$ in $\bar{\Omega}_{0}$ other than $\overline{\xi_{i} \xi_{j}}$ is either $\bar{\xi}_{k}$ or $\bar{\xi}_{l}$, say $\bar{\xi}_{k}$. However, by the Q-argument with $Q=A B \bar{\xi}_{k} \xi_{l} \bar{\xi}_{k}$, the configuration $\Gamma$ is 1-reducible.

Now we prove statement 3$)$. By statement 1$)$, we may assume that $d\left(B, \bar{\Omega}_{0}\right)=d\left(C, \bar{\Omega}_{0}\right)$ $=2$. If $D \in N\left(B, \bar{\Omega}_{0}\right) \cap N\left(C, \bar{\Omega}_{0}\right)$, then the Q-argument with $Q=D B A C$ shows that the configuration $\Gamma$ is 1-reducible. Thus, applying (22) to $B$ and $C$ and using the T-argument, we see that at least one of the sets $N\left(B, \bar{\Omega}_{0}\right), N\left(C, \bar{\Omega}_{0}\right)$, say the first set, is of the form $N\left(B, \bar{\Omega}_{0}\right)=\left\{\bar{\xi}_{i} \xi_{j}, \bar{\xi}_{k} \xi_{l}\right\}$, where $i, j, k, l$ are pairwise distinct. From the hypothesis of the statement it follows that there exists $\xi \in \Omega_{0}$ such that $\bar{\xi} \in N\left(A, \bar{\Omega}_{0}\right)$. The T-argument shows that $\xi \in \Xi$. Put $D=\bar{\xi}_{i} \xi_{j}$ if $\xi \in\left\{\xi_{i}, \xi_{j}\right\}$, and $D=\bar{\xi}_{k} \xi_{l}$ if $\xi \in\left\{\xi_{k}, \xi_{l}\right\}$. Then, by the Q-argument with $Q=A \bar{\xi} D B$, the configuration $\Gamma$ is 1-reducible.

\section{§8. Proof of Theorem 7.4}

Given $\xi \in \Sigma$, we set $\xi^{\prime}=\xi^{f}$, where $f=\left(\xi_{1}, \xi_{2}\right)\left(\xi_{3}, \xi_{4}\right)(\sigma, \tau) \in \operatorname{Sym}(\Sigma)$; if $\xi=\eta \zeta$ with $\eta, \zeta \in \Sigma$, then, by definition, $\xi^{\prime}=\eta^{\prime} \zeta^{\prime}$. The element $\xi^{\prime}$ is called the twin of $\xi$. In what follows, by convention, given a configuration $\Gamma$ on $\Omega$, we set $\bar{\xi}=\varnothing$ whenever $\xi \in \Omega \backslash \Omega$. Concerning the notation $X$ and $X^{*}$, see (21).

Lemma 8.1. Suppose that an admissible configuration $\Gamma$ is neither final nor 1-reducible. Then $X^{*} \neq \varnothing$ and $d(A)=3$ for each $A \in X^{*}$. Moreover, exactly one of the following two statements is true:

I) for all $v \in\{\sigma, \tau\}$ and $\xi \in \Xi$ such that $A=\overline{v \xi} \in X^{*}$, we have $N(A)=\{\bar{\xi}, \bar{\sigma}, \bar{\tau}\}$;

II) for all $v \in\{\sigma, \tau\}$ and $\xi \in \Xi$ such that $A=\overline{v \xi} \in X^{*}$, we have $N(A)=\left\{\bar{\xi}, \bar{v}, A^{\prime}\right\}$, where $A^{\prime}$ is the class containing the twin of $v \xi$.

Proof. Suppose $X^{*}=\varnothing$. Then all elements of $\bar{\Omega}$ are pairwise comparable. Since $\Gamma$ is not final, the set $X$ is not empty. So, by the D-argument, without loss of generality we may assume that $d(A, \bar{\Omega}) \geq 3$ for all $A \in X$. In particular, if $d(A, X)=0$ for some $A \in X$, then the configuration $\Gamma$ is 1-reducible by statement 1) of Lemma 7.8, a contradiction. Otherwise $|X| \geq 2$ and $d(A, X) \geq 1$ for all $A \in X$. If $|X|=2$, then $\Gamma$ is 1-reducible by 
statement 2) of Lemma 7.8 a contradiction. Thus, $|X|=3$ and $X=\{\bar{\sigma}, \bar{\tau}, \overline{\sigma \tau}\}$. Then $N(\overline{\sigma \tau})=\{\bar{\sigma}, \bar{\tau}\} \cup N\left(\overline{\sigma \tau}, \overline{\Omega_{0}}\right)$ by the T-argument. So, the configuration $\Gamma$ is 1-reducible by the D-argument with $A=\overline{\sigma \tau}$ if $d\left(\overline{\sigma \tau}, \overline{\Omega_{0}}\right)=0$, and by statement 3) of Lemma 7.8 with $A=\overline{\sigma \tau}, B=\bar{\sigma}$, and $C=\bar{\tau}$ if $d\left(\overline{\sigma \tau}, \overline{\Omega_{0}}\right)>0$. Again we arrive at a contradiction. Thus, $X^{*} \neq \varnothing$.

Let $A=\overline{v \xi} \in X^{*}$, where $v \in\{\sigma, \tau\}$ and $\xi \in \Xi$. Then $A \subset \Omega_{1}^{*} \cup \Omega_{2}^{*}$. Without loss of generality we may assume that $v=\sigma$ and $\xi=\xi_{1}$. Since the configuration $\Gamma$ is admissible, we have $N(A) \subset \bar{\Omega}_{1}$. So, by the T-argument, we conclude that

$$
N(A) \subset\left\{A^{\prime}, \bar{\xi}_{1}, \bar{\xi}_{2}, \bar{\sigma}, \bar{\tau}\right\} .
$$

If $\bar{\xi}_{1} \neq \bar{\xi}_{2}$ and $\bar{\xi}_{2} \in N(A)$, then the configuration $\Gamma$ is 1-reducible by the Q-argument with $Q=\bar{\xi}_{1} A \bar{\xi}_{2} \bar{\xi}_{1} \xi_{2}$, a contradiction. Thus, $N(A) \subset\left\{A^{\prime}, \bar{\xi}_{1}, \bar{\sigma}, \bar{\tau}\right\}$. By the T-argument we conclude that either $N(A) \subset\left\{\bar{\xi}_{1}, \bar{\sigma}, \bar{\tau}\right\}$ or $N(A) \subset\left\{\bar{\xi}_{1}, \bar{\sigma}, A^{\prime}\right\}$. In any case the elements of $N(A)$ are pairwise comparable. Since $\Gamma$ is not 1-reducible, the D-argument implies that $d(A)=3$. In particular, the neighborhood of each class in $X^{*}$ is of type I or of type II. If there are no neighborhoods of type I, we are done. Otherwise, we have $\sigma, \tau \in \Omega$ and

$$
t_{1}(\sigma)=t_{1}(\tau) .
$$

In this case, if there are no neighborhoods of type II, then we are done. Otherwise, there exists a class $A=\overline{v \xi} \in X^{*}$ with neighborhood of type II. Since the class $A^{\prime}$ contains the element $v^{\prime} \xi^{\prime}$, we have $t_{1}(v)=t_{1}\left(v^{\prime} \xi^{\prime}\right) \neq t_{1}\left(v^{\prime}\right)$ (T-argument). Since $\left\{v, v^{\prime}\right\}=\{\sigma, \tau\}$, this implies that $t_{1}(\sigma) \neq t_{1}(\tau)$, which contradicts (23). Thus, exactly one of statements I), II) is true.

Now we turn to the proof of Theorem 7.4. Let $\Gamma$ be a nonfinal admissible configuration. There is no loss of generality in assuming that $\Gamma$ is not 1-reducible. Then, by Lemma 8.1, we have $X^{*} \neq \varnothing$ and $d(A)=3$ for all $A \in X^{*}$. We consider two cases, depending on which particular statement, I) or II), of that lemma holds true.

Case I. In this case, $\sigma, \tau \in \Omega$ and $t_{1}(\sigma)=t_{1}(\tau)$. Consider two possibilities.

Suppose $\left|X^{*}\right|=1$. Then $X^{*}=\{A\}$ and $N(A)=\{\bar{\xi}, \bar{\sigma}, \bar{\tau}\}$, where $A=\overline{v_{0}} \xi, v_{0} \in\{\sigma, \tau\}$, and $\xi \in \Xi$. So, $\sigma \tau \notin \Omega$, because otherwise, by the Q-argument with $Q=\bar{\sigma} A \bar{\tau} \overline{\sigma \tau}$, the configuration $\Gamma$ is 1-reducible ( $\sigma \tau$ is incident to both $\sigma$ and $\tau$ by the T-argument). We have arrived at a contradiction. Therefore, $N(\bar{v}) \subset \bar{\Omega}_{0} \cup\{A\}$, where $v \in\{\sigma, \tau\}$. If $\bar{v} \in X$, then $d\left(\bar{v}, \bar{\Omega}_{0}\right) \geq 2$ by the $\mathrm{D}$-argument. Thus, we get a contradiction with statement 1 ) of Lemma 7.8 if $\bar{\sigma}, \bar{\tau} \in \bar{\Omega}_{0}$, with statement 2) if only one of these elements, say $\bar{\sigma}$, belongs to $\bar{\Omega}_{0}(B=\bar{\sigma})$, and with statement 3$)$ if $\bar{\sigma}, \bar{\tau} \notin \bar{\Omega}_{0}(B=\bar{\sigma}, C=\bar{\tau})$.

Suppose $\left|X^{*}\right| \geq 2$. In $X^{*}$ we take two distinct classes $A_{1}=\overline{v_{1} \xi}$ and $A_{2}=\overline{v_{2} \eta}$, where $v_{1}, v_{2} \in\{\sigma, \tau\}$ and $\xi, \eta \in \Xi$. Then $N\left(A_{1}\right)=\{\bar{\xi}, \bar{\sigma}, \bar{\tau}\}$ and $N\left(A_{2}\right)=\{\bar{\eta}, \bar{\sigma}, \bar{\tau}\}$. If $\bar{\xi}=\bar{\eta}$, then $N\left(A_{1}\right)=N\left(A_{2}\right)$. By statement 1) of Lemma 7.6, this shows that $A_{1}$ and $A_{2}$ are comparable. So, by the Q-argument with $Q=\bar{\sigma} A_{1} \bar{\tau} A_{2}$, the configuration $\Gamma$ is 1-reducible. We have arrived at a contradiction. Thus, $\bar{\xi} \neq \bar{\eta}$. Then, by statement 2) of Lemma [7.6] with $P=A_{1} \bar{\xi} \overline{\xi \eta} \bar{\eta} A_{2}$, the classes $A_{1}$ and $A_{2}$ are quasicomparable. Now the generalized Q-argument with $Q=\bar{\sigma} A_{1} \bar{\tau} A_{2}$ shows that the configuration $\Gamma$ is 2-reducible, and we are done.

Case II. In this case, the T-argument implies that $\tau \sigma \notin \Omega$ and if $\sigma, \tau \in \Omega$, then $t_{1}(\sigma) \neq t_{1}(\tau)$. Furthermore, the following is true.

Lemma 8.2. Suppose $\overline{v \xi} \in X^{*}$, where $v \in\{\sigma, \tau\}$ and $\xi \in \Xi$. Then:

1) $\bar{v} \in X$

2) $d(\bar{v})=d\left(\bar{v}, X^{*}\right)=2$, and $N(\bar{v})=\{\overline{v \xi}, \overline{v \eta}\}$ for some $\eta \in \Xi$;

3) $t_{1}(\xi)=t_{1}(\eta)=t_{1}(v), t_{1}\left(\xi^{\prime}\right)=t_{1}\left(\eta^{\prime}\right) \neq t_{1}(v)$. 
S. EVDOKIMOV AND I. PONOMARENKO

Proof. Without loss of generality we assume that $v=\sigma$ and $\xi=\xi_{1}$. Set $A=\overline{v \xi}$. By Lemma 8.1] $d(A)=3$ and $N(A)=\left\{\bar{\xi}_{1}, \bar{\sigma}, A^{\prime}\right\}$ with $A^{\prime}=\bar{\tau}_{2}$. In particular, $t_{1}\left(\xi_{1}\right) \neq$ $t_{1}\left(\xi_{2}\right)$. Moreover, if $\tau \in \Omega$, then, by the Q-argument with $Q=\bar{\sigma} \bar{\tau} A^{\prime} A$, the classes $\sigma$ and $\tau$ are not incident.

Suppose that $\bar{\sigma} \in \bar{\Omega}_{0}$. Then by (22) we have $\bar{\sigma}=\overline{\xi_{j} \xi_{k}}$ for distinct $j, k \in\{2,3,4\}$. Moreover, $j, k \neq 2$ by the Q-argument with $Q=A \bar{\sigma} \bar{\xi}_{2} A^{\prime}$. Thus, $\bar{\sigma}=\overline{\xi_{3} \xi_{4}}$. Also, by statement 1) of Lemma 7.8 we have $A^{\prime} \notin \bar{\Omega}_{0}$. So, $A^{\prime} \in X^{*}$, because otherwise $A^{\prime}=\bar{\tau}$ and $t_{1}\left(A^{\prime}\right)=t_{1}(\sigma) \neq t_{1}(\tau)$ in contrast to the T-argument. Therefore, by Lemma 8.1 , we have $\tau \in \Omega$ and $\bar{\tau} \neq \bar{\xi}_{2}$. Applying statement 2) of Lemma 7.8 with $B=A^{\prime}$, we see that $\bar{\tau} \notin \bar{\Omega}_{0}$. Since $t_{1}\left(\xi_{1}\right) \neq t_{1}\left(\xi_{2}\right)=t_{1}\left(\xi_{3}\right)=t_{1}\left(\xi_{4}\right)$, it follows that

$$
N\left(\bar{\tau}, \bar{\Omega}_{0}\right) \subset\left\{\bar{\xi}_{1}, \overline{\xi_{2} \xi_{3}}, \overline{\xi_{2} \xi_{4}}, \overline{\xi_{3} \xi_{4}}\right\} .
$$

So, the Q-argument with $Q=A A^{\prime} \bar{\tau} \bar{\xi}_{1}, Q=\bar{\tau} A^{\prime} \bar{\xi}_{2} \overline{\xi_{2} \xi_{3}}, Q=\bar{\tau} A^{\prime} \bar{\xi}_{2} \overline{\xi_{2} \xi_{4}}$, and $Q=$ $A A^{\prime} \bar{\tau} \bar{\sigma}$ shows that $N\left(\bar{\tau}, \bar{\Omega}_{0}\right)=\varnothing$. Since the classes $\bar{\sigma}$ and $\bar{\tau}$ are not incident, this implies that $N(\bar{\tau}) \subset X^{*}$, whence

$$
N(\bar{\tau}) \subset X^{*} \cap\left\{\overline{\tau \xi}_{2}, \overline{\tau \xi}_{3}, \overline{\tau \xi}_{4}\right\}
$$

(T-argument). From the D-argument it follows that $d(\bar{\tau})>1$, so that $\overline{\tau \xi}_{i} \in N(\bar{\tau})$ for some $i \in\{3,4\}$, say $i=3$. Since $\overline{\tau \xi_{3}} \in X^{*}$, we conclude that $\overline{\sigma \xi_{4}} \in N\left(\overline{\tau \xi_{3}}\right)$ by Lemma 8.1, which contradicts the fact that $t_{1}\left(\xi_{3}\right)=t_{1}\left(\xi_{4}\right)$. This proves statement 1$)$.

Suppose that $d\left(\bar{\sigma}, X^{*}\right)<1$. Then, since $\bar{\tau} \notin N(\bar{\sigma})$, the D-argument with $A=\bar{\sigma}$ implies that $d\left(\bar{\sigma}, \bar{\Omega}_{0}\right) \geq 2$. Since $t_{1}\left(\xi_{1}\right) \neq t_{1}\left(\xi_{2}\right)$, formula (22) yields $N\left(\bar{\sigma}, \bar{\Omega}_{0}\right) \supset\left\{\bar{\xi}_{i}, \overline{\xi_{j} \xi_{k}}\right\}$, where $i, j, k$ are pairwise distinct elements of $\{1,2,3,4\}, i \neq 1, j, k \neq 2$, and $\bar{\xi}_{i} \neq \bar{\xi}_{j} \xi_{k}$. However, $i \neq 2$ (respectively, $j \neq 1, k \neq 1$ ) by the Q-argument with $Q=A A^{\prime} \bar{\xi}_{2} \bar{\sigma}$ (respectively, $Q=A \bar{\sigma} \bar{\xi}_{1} \bar{\xi}_{k} \bar{\xi}_{1}, Q=A \bar{\sigma} \bar{\xi}_{1} \bar{\xi}_{j} \bar{\xi}_{1}$ ). So, $i, j, k \in\{3,4\}$, a contradiction. Thus, $d\left(\bar{\sigma}, X^{*}\right) \geq 2$. Therefore, there exists $B \in N\left(\bar{\sigma}, X^{*}\right)$ other than $A$. Then by the T-argument we have $B \in\left\{\overline{\sigma \xi_{3}}, \overline{\sigma \xi_{4}}\right\}$, say $B=\overline{\sigma \xi_{3}}$. In this case, Lemma 8.1 implies that $N(B)=\left\{B^{\prime}, \bar{\sigma}, \bar{\xi}_{3}\right\}$ with $B^{\prime}=\overline{\tau \xi}_{4}$. So,

$$
t_{1}\left(\xi_{1}\right)=t_{1}\left(\xi_{3}\right)=t_{1}(\sigma), \quad t_{1}\left(\xi_{2}\right)=t_{1}\left(\xi_{4}\right) \neq t_{1}(\sigma),
$$

which proves statement 3$)$. This also shows that $N\left(\bar{\sigma}, X^{*}\right)=\{A, B\}$ and $N\left(\bar{\sigma}, \bar{\Omega}_{0}\right) \subset$ $\left\{\overline{\xi_{1} \xi_{3}}, \bar{\xi}_{2}, \bar{\xi}_{4}\right\}$ (T-argument). However, from the Q-argument with $Q=A \bar{\sigma} \overline{\xi_{1} \xi_{3}} \bar{\xi}_{1}$ (respectively, $Q=A A^{\prime} \bar{\xi}_{2} \bar{\sigma}, Q=B B^{\prime} \bar{\xi}_{4} \bar{\sigma}$ ) it follows that $\bar{\xi}_{1} \xi_{3}$ (respectively, $\bar{\xi}_{2}, \bar{\xi}_{4}$ ) does not belong to $N\left(\bar{\sigma}, \bar{\Omega}_{0}\right)$. Thus, $N(\bar{\sigma})=\{A, B\}$.

Since $X^{*} \neq \varnothing$, statement 2) of Lemma 8.2 shows that $X^{*}$ contains distinct classes $A_{1}=\overline{v \xi}$ and $A_{2}=\overline{v \eta}$, where $v \in\{\sigma, \tau\}$ and $\xi, \eta \in \Xi$, such that $\bar{v} \in X$ and $N(\bar{v})=$ $\left\{A_{1}, A_{2}\right\}$. Moreover, $N\left(A_{1}\right)=\left\{\bar{\xi}, \bar{v}, A_{1}^{\prime}\right\}$ with $A_{1}^{\prime}=\overline{v^{\prime} \xi^{\prime}}$ and $N\left(A_{2}\right)=\left\{\bar{\eta}, \bar{v}, A_{2}^{\prime}\right\}$ with $A_{2}^{\prime}=\overline{v^{\prime} \eta^{\prime}}$. Without loss of generality we may assume that

$$
\bar{\xi}=\bar{\eta} \text {. }
$$

(Otherwise, by statement 2) of Lemma 7.6 with $P=A_{1} \bar{\xi} \overline{\xi \eta} \bar{\eta} A_{2}$, the classes $A_{1}$ and $A_{2}$ are quasicomparable. Then by the generalized D-argument with $A=\bar{v}$, the configuration $\Gamma$ is 2-reducible and we are done.) To complete the proof, we consider two cases.

Suppose $X^{*}=\left\{A_{1}, A_{2}\right\}$. Then $A_{1}^{\prime}, A_{2}^{\prime} \in \bar{\Omega}_{0}$. Applying (24) and statement 3) of Lemma 8.2, we see that $A_{1}^{\prime}=\overline{\xi^{\prime} \eta^{\prime}}=A_{2}^{\prime}$ (T-argument). So, $N\left(A_{1}\right)=N\left(A_{2}\right)$, whence $A_{1}$ and $A_{2}$ are comparable by statement 1 ) of Lemma 7.6. Now the D-argument with $A=\bar{v}$ shows that the configuration $\Gamma$ is 1-reducible, a contradiction.

Suppose $X^{*} \neq\left\{A_{1}, A_{2}\right\}$. By Lemma 8.2 (applied to an element of $X^{*}$ other than $A_{1}$ and $\left.A_{2}\right)$ we have $X^{*}=\left\{A_{1}, A_{2}, A_{1}^{\prime}, A_{2}^{\prime}\right\}$ and $N\left(\overline{v^{\prime}}\right)=\left\{A_{1}^{\prime}, A_{2}^{\prime}\right\}$, where $A_{1}^{\prime} \neq A_{2}^{\prime}$ 
and $\bar{v}^{\prime} \in X$. Moreover, $N\left(A_{1}^{\prime}\right)=\left\{\bar{\xi}^{\prime}, \bar{v}^{\prime}, A_{1}\right\}$ and $N\left(A_{2}^{\prime}\right)=\left\{\bar{\eta}^{\prime}, \bar{v}^{\prime}, A_{2}\right\}$. By (24) (with $A_{1}=A_{1}^{\prime}$ and $\left.A_{2}=A_{2}^{\prime}\right)$, we may assume that $\bar{\xi}^{\prime}=\bar{\eta}^{\prime}$. Then

$$
\begin{aligned}
& N\left(A_{1}\right) \backslash\left\{A_{1}^{\prime}\right\}=\{\bar{v}, \bar{\xi}\}=N\left(A_{2}\right) \backslash\left\{A_{2}^{\prime}\right\}, \\
& N\left(A_{1}^{\prime}\right) \backslash\left\{A_{1}\right\}=\left\{\bar{v}^{\prime}, \bar{\xi}^{\prime}\right\}=N\left(A_{2}^{\prime}\right) \backslash\left\{A_{2}\right\} .
\end{aligned}
$$

Therefore, by statement 2) of Lemma 7.6 with $P=A_{1} A_{1}^{\prime} \bar{\xi}^{\prime} A_{2}^{\prime} A_{2}$, the classes $A_{1}$ and $A_{2}$ are quasicomparable. Thus, by the generalized D-argument with $A=\bar{v}$, the configuration $\Gamma$ is 2-reducible.

\section{§9. Proof of Theorem 1.2}

Let $\Gamma=(\Omega, E, t)$ be a configuration, where $t=\left(t_{1}, t_{2}\right)$. Given $(\xi, \eta) \in E$, we set $t(\xi, \eta)=\left(t_{1}(\xi), t_{2}(\xi, \eta)\right)$; a pair $\left(\mathfrak{t}_{1}, \mathfrak{t}_{2}\right) \in \mathfrak{T}$, where $\mathfrak{T}=\mathfrak{T}_{1} \times \mathfrak{T}_{2}$, is denoted by $\left(\mathfrak{t}_{2}\right)_{\mathfrak{t}_{1}}$ (see the notation). From now on, we assume that $\Gamma$ is either the configuration corresponding to a projective plane (see the beginning of 4 ) or a $\Xi$-configuration. Then for some pairs of its elements we can consider the product of them, which is also an element of $\Gamma$. It is convenient to set the value of the function $t$ to be $\varnothing$ if one of the arguments of $t$ is an "undefined" product.

Let $V$ be the set of elements of a projective plane $\mathcal{P}$. For $i, j, k, l \in\{1,2,3,4\}$, we set

$$
\begin{gathered}
\operatorname{Cyl}_{\mathfrak{t}}(i, j)=\left\{x \in V^{4}: t\left(x_{i}, x_{j}\right)=\mathfrak{t}\right\}, \quad \mathfrak{t} \in \mathfrak{T}, \\
\operatorname{Col}_{\mathfrak{s}}(i, j, k)=\left\{x \in V^{4}: t\left(x_{i}, x_{j} x_{k}\right)=t\left(x_{j}, x_{k} x_{i}\right)=t\left(x_{k}, x_{i} x_{j}\right)=\mathfrak{i}_{\mathfrak{s}}\right\}, \quad \mathfrak{s} \in \mathfrak{T}_{1}, \\
\operatorname{Col}_{\mathfrak{s}}^{(2)}(i, j, k, l)=\left\{x \in V^{4}: t\left(x_{i} x_{j}, x_{k} x_{l}\right)=\mathfrak{i}_{\mathfrak{s}^{\prime}}, t\left(x_{i}, x_{k} x_{l}\right)=t\left(x_{j}, x_{k} x_{l}\right)=\mathfrak{e}_{\mathfrak{s}}^{\prime},\right. \\
\left.t\left(x_{k}, x_{i} x_{j}\right)=t\left(x_{l}, x_{i} x_{j}\right)=\mathfrak{e}_{\mathfrak{s}^{\prime}}^{\prime}\right\}, \quad \mathfrak{s} \in \mathfrak{T}_{1},
\end{gathered}
$$

where $t=\left(t_{1, \mathcal{P}}, t_{2, \mathcal{P}}\right)$. We observe that $\mathrm{Cyl}_{\mathfrak{t}}(i, j)=\mathrm{Cyl}_{R}(i, j)$, where $R$ equals $E_{\mathfrak{s}}$ (respectively, $\left.E_{\mathfrak{s}}^{\prime}, I_{\mathfrak{s}}, I_{\mathfrak{s}}^{\prime}\right)$ for $\mathfrak{t}=e_{\mathfrak{s}}$ (respectively, $\left.e_{\mathfrak{s}}^{\prime}, i_{\mathfrak{s}}, i_{\mathfrak{s}}^{\prime}\right), \mathfrak{s} \in \mathfrak{T}_{1}$ (see (11), (2) and (33). Next, by the above convention, if $x \in \operatorname{Col}_{\mathfrak{s}}(i, j, k)$, then $t_{1}\left(x_{i}\right)=t_{1}\left(x_{j}\right)=t_{1}\left(x_{k}\right)=\mathfrak{s}$, and the elements $x_{i}, x_{j}, x_{k}$ (and, thus, the indices $\left.i, j, k\right)$ are pairwise distinct. It is easily seen that the relation given by (26) can be rephrased by demanding that the elements $x_{i}, x_{j}, x_{k}$ be collinear 5 Similarly, if $x \in \operatorname{Col}_{\mathfrak{s}}^{(2)}(i, j, k, l)$, then $t_{1}\left(x_{i}\right)=t_{1}\left(x_{j}\right)=\mathfrak{s}$ and $t_{1}\left(x_{k}\right)=t_{1}\left(x_{l}\right)=\mathfrak{s}^{\prime}$, where $\mathfrak{s}^{\prime}$ is the type dual to $\mathfrak{s}$. Moreover, the elements $x_{i}, x_{j}, x_{k}, x_{l}$, and so the indices $i, j, k, l$, are pairwise distinct. In fact, the relation given by (27) can also be rephrased by demanding that the elements $x_{i}, x_{j}, x_{k} x_{l}$ as well as the elements $x_{k}$, $x_{l}, x_{i} x_{j}$ be collinear and pairwise distinct. The relations defined in (25), (26) and (27) are called the cylindricity, collinearity, and double collinearity relations of the plane $\mathcal{P}$.

Lemma 9.1. The derivative partition $\Pi=\operatorname{Der}(\mathcal{P})$ associated with the plane $\mathcal{P}$ coincides with the partition of $V^{4}$ generated by all cylindricity, collinearity, and double collinearity relations of the plane $\mathcal{P}$, i.e., with the coarsest partition of $V^{4}$ for which any of these relations is a union of classes.

Proof. Let $\Pi^{\prime}$ denote the second partition occurring in the lemma. It is easily seen that for any $i, j, k, l \in\{1,2,3,4\}$ and $\mathfrak{t} \in \mathfrak{T}$ (respectively, $\mathfrak{s} \in \mathfrak{T}_{1}$ ) the relation $\mathrm{Cyl}_{\mathfrak{t}}(i, j$ ) (respectively, $\left.\operatorname{Col}_{\mathfrak{s}}(i, j, k), \mathrm{Col}_{\mathfrak{s}}^{(2)}(i, j, k, l)\right)$ is the union of the relations $R(\Gamma, \mathcal{P})$, where $\Gamma$ runs over all configurations belonging to the classes $\mathcal{K}_{0}$ (respectively, $\mathcal{K}_{1}$ and $\mathcal{K}_{2}$ ) defined

\footnotetext{
${ }^{5}$ Elements of $\mathcal{P}$ are said to be collinear if there exists an element incident to all of them; thus, concurrent lines are also called collinear here.
} 
as follows:

$$
\begin{aligned}
& \mathcal{K}_{0}=\left\{\Gamma \in \mathcal{K}: t\left(\xi_{i}, \xi_{j}\right)=\mathfrak{t}\right\}, \\
& \mathcal{K}_{1}=\left\{\Gamma \in \mathcal{K}: t\left(\xi_{i}, \xi_{j} \xi_{k}\right)=t\left(\xi_{j}, \xi_{k} \xi_{i}\right)=t\left(\xi_{k}, \xi_{i} \xi_{j}\right)=\mathfrak{i}_{\mathfrak{s}}\right\}, \\
& \mathcal{K}_{2}=\left\{\Gamma \in \mathcal{K}: t\left(\xi_{i} \xi_{j}, \xi_{k} \xi_{l}\right)=\mathfrak{i}_{\mathfrak{s}^{\prime}}, t\left(\xi_{i}, \xi_{k} \xi_{l}\right)=t\left(\xi_{j}, \xi_{k} \xi_{l}\right)=\mathfrak{e}_{\mathfrak{s}}^{\prime},\right. \\
&\left.\quad t\left(\xi_{k}, \xi_{i} \xi_{j}\right)=t\left(\xi_{l}, \xi_{i} \xi_{j}\right)=\mathfrak{e}_{\mathfrak{s}^{\prime}}^{\prime}\right\} ;
\end{aligned}
$$

here $\mathcal{K}$ is the class of all $\Xi$-configurations. Thus, $\Pi$ is coarser than $\Pi^{\prime}$. Conversely, let $R \in \Pi$. Then $R=R(\Gamma, \mathcal{P})$ for some $\Xi$-configuration $\Gamma=\left(\Omega, \Omega^{2}, t\right)$. It is easily seen that

$$
R=\bigcap_{\xi, \eta \in \Omega} R_{\xi, \eta, t(\xi, \eta)}
$$

where for $\mathfrak{t} \in \mathfrak{T}$ and $\xi, \eta \in \Omega$ we have set

$$
R_{\xi, \eta, \mathfrak{t}}=\left\{x \in V^{4}: \xi, \eta \in \Omega_{x}, t_{x}(\xi, \eta)=\mathfrak{t}\right\}
$$

with $\Omega_{x}$ and $t_{x}$ such that $\operatorname{Der}(x, \mathcal{P})=\left(\Omega_{x}, \Omega_{x}^{2}, t_{x}\right)$ (see the end of $\varangle 4$ ). So, it suffices to verify that any relation $R_{\xi, \eta, t}$ is a union of elements of the partition $\Pi^{\prime}$.

Obviously, if $\xi=\xi_{i}$ and $\eta=\xi_{j}$ for some $i, j \in\{1, \ldots, 4\}$, then $R_{\xi, \eta, \mathfrak{t}}=\operatorname{Cyl}_{\mathfrak{t}}(i, j)$. Let $\xi=\xi_{i}$ and $\eta=\xi_{j} \xi_{k}$ for some $i, j, k \in\{1, \ldots, 4\}$ (the case where $\xi=\xi_{j} \xi_{k}$ and $\eta=\xi_{i}$ is treated similarly). Then

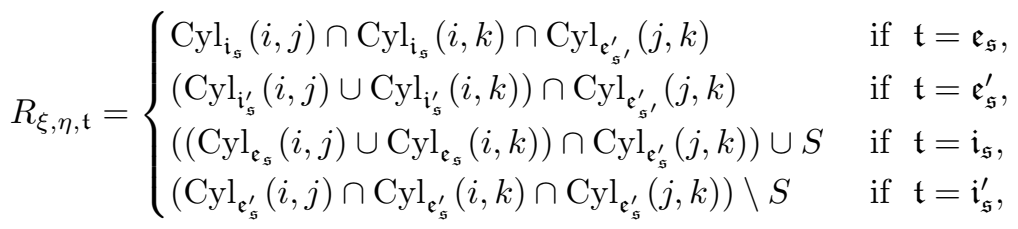

where $\mathfrak{s} \in \mathfrak{T}_{1}$ and $S=\operatorname{Col}_{\mathfrak{s}}(i, j, k)$ if $i, j, k$ are pairwise distinct, and $S=\varnothing$ otherwise. Finally, if $\xi=\xi_{i} \xi_{j}$ and $\eta=\xi_{k} \xi_{l}$, then

$$
R_{\xi, \eta, \mathfrak{t}}= \begin{cases}R_{\xi_{i}, \eta, \mathfrak{i}_{\mathfrak{s}^{\prime}}} \cap R_{\xi_{j}, \eta, \mathfrak{i}_{\mathfrak{s}^{\prime}}} & \text { if } \mathfrak{t}=\mathfrak{e}_{\mathfrak{s}}, \\ \left(\mathrm{Cyl}_{\mathfrak{e}^{\prime}}(i, j) \cap \mathrm{Cyl}_{\mathfrak{e}_{\mathfrak{s}}^{\prime}}(i, k)\right) \backslash R_{\xi, \eta, \mathfrak{e}_{\mathfrak{s}}} & \text { if } \mathfrak{t}=\mathfrak{e}_{\mathfrak{s}}^{\prime}, \\ \operatorname{Col}_{\mathfrak{t}}(2)(i, j, k, l) \cup S_{1} \cup S_{2} \cup S_{3} \cup S_{4} & \text { if } \mathfrak{t}=\mathfrak{i}_{\mathfrak{s}}, \\ \left(\operatorname{Cyl}_{\mathfrak{e}_{\mathfrak{s}}^{\prime}}(i, j) \cap \mathrm{Cyl}_{\mathfrak{e}_{\mathfrak{s}^{\prime}}^{\prime}}(i, k)\right) \backslash R_{\xi, \eta, i_{\mathfrak{s}}} & \text { if } \mathfrak{t}=\mathfrak{i}_{\mathfrak{s}}^{\prime},\end{cases}
$$

where $\mathfrak{s} \in \mathfrak{T}_{1}$ and $S_{1}=R_{\xi_{i}, \eta, \mathfrak{e}_{\mathfrak{s}}} \cap \mathrm{Cyl}_{\mathfrak{e}_{\mathfrak{s}}^{\prime}}(i, j), S_{2}=R_{\xi_{j}, \eta, \mathfrak{e}_{\mathfrak{s}}} \cap \mathrm{Cyl}_{\mathfrak{e}_{\mathfrak{s}}^{\prime}}(i, j), S_{3}=R_{\xi, \xi_{k}, \mathfrak{e}_{\mathfrak{s}^{\prime}}} \cap$ $\mathrm{Cyl}_{\mathfrak{e}_{\mathfrak{s}^{\prime}}}(k, l), S_{4}=R_{\xi, \xi_{l}, \mathfrak{e}_{\mathfrak{s}^{\prime}}} \cap \mathrm{Cyl}_{{\mathfrak{\mathfrak { s } ^ { \prime }}}_{\mathfrak{s}^{\prime}}}(k, l)$.

We turn to the proof of Theorem[1.2. Let $\mathcal{C}^{\prime}=\left(V^{2}, \Pi\right)$ be the derivative scheme of $\mathcal{P}$. It is easily seen that

$\Delta\left(\Delta_{2}\right)=\bigcup_{\mathfrak{s} \in \mathfrak{T}_{1}}\left(\operatorname{Cyl}_{\mathfrak{e}_{\mathfrak{s}}}(1,2) \cap \mathrm{Cyl}_{\mathfrak{e}_{\mathfrak{s}}}(1,3) \cap \mathrm{Cyl}_{\mathfrak{e}_{\mathfrak{s}}}(1,4)\right), \quad R \otimes S=\mathrm{Cyl}_{R}(1,3) \cap \mathrm{Cyl}_{S}(2,4)$,

where $\Delta_{2}=\Delta(V)$ and $R, S$ are basis relations of the scheme $\mathcal{C}$. Therefore, by Lemma 9.1 and the definition of the 2-extension (see (10)), we have

$$
\mathcal{C}^{\prime} \geq \widehat{\mathcal{C}} .
$$

By Lemma 9.1, to prove the reverse inclusion it suffices to verify that all cylindricity, collinearity, and double collinearity relations of the plane $\mathcal{P}$ are relations of the scheme $\hat{\mathcal{C}}$. We note that the statement on the cylindricity relations follows from Theorem 3.2 .

To prove the statement about the collinearity relations, suppose $i, j, k \in\{1, \ldots, 4\}$ are pairwise distinct and $\mathfrak{s} \in \mathfrak{T}_{1}$. We observe that $\operatorname{Col}_{\mathfrak{s}}(i, j, k)$ does not change under any permutation of $i, j, k$. So, we can assume that $\{i, j\}=\{1,2\}$ or $\{i, j\}=\{3,4\}$. On the other hand, $\operatorname{Col}_{\mathfrak{s}}(3,4, k)^{T}=\operatorname{Col}_{\mathfrak{s}}\left(1,2, k^{\prime}\right)$, where $k^{\prime}=k+2$, and $\mathcal{R}^{*}(\widehat{\mathcal{C}})^{T}=\mathcal{R}^{*}(\widehat{\mathcal{C}})$. Then 
without loss of generality we may assume that $\{i, j\}=\{1,2\}$. However, in this case we have

$$
\mathrm{Col}_{\mathfrak{s}}(i, j, k)=\left(S_{1} \cap S_{2}\right) \cdot \operatorname{Cyl}_{\mathfrak{e}_{\mathfrak{s}}}(k-2, k),
$$

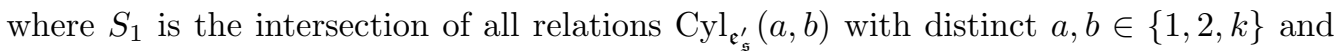
$S_{2}$ is the intersection of all relations $\operatorname{Cyl}_{\mathfrak{i}_{\mathrm{s}}}\left(a, k^{\prime}\right)^{\mathrm{s}}$ with $a \in\{1,2, k\}$, and $k^{\prime}$ is uniquely determined by the condition $\left\{k, k^{\prime}\right\}=\{3,4\}$. Thus, the required statement follows from the already proved statement that any cylindricity relation is a relation of the scheme $\hat{\mathcal{C}}$.

To prove the statement about the double collinearity relations, suppose $\{i, j, k, l\}=$ $\{1,2,3,4\}$ and $\mathfrak{s} \in \mathfrak{T}_{1}$. We note that $\operatorname{Col}_{\mathfrak{s}}^{(2)}(i, j, k, l)$ does not change under any permutation of the numbers $i, j$ as well as the numbers $k, l$. This implies that if $\{i, j\} \cap\{1,2\} \neq \varnothing$, then either $\{i, j\}=\{1,2\}$, or without loss of generality we may assume that $\{i, k\}=$ $\{1,2\}$. On the other hand, if $\{i, j\}=\{3,4\}$, then $\mathrm{Col}_{\mathfrak{s}}^{(2)}(i, j, k, l)^{T}=\mathrm{Col}_{\mathfrak{s}^{\prime}}^{(2)}(k, l, i, j)$. Therefore, since $\mathcal{R}^{*}(\widehat{\mathcal{C}})^{T}=\mathcal{R}^{*}(\widehat{\mathcal{C}})$, in any case we can assume that $\{i, j\}=\{1,2\}$ or $\{i, k\}=\{1,2\}$. But then a straightforward check shows that

$$
\operatorname{Col}_{\mathfrak{s}}^{(2)}(i, j, k, l)= \begin{cases}\left(S_{1} \cdot S_{2}\right) \cap \mathrm{Cyl}_{\mathfrak{e}_{\mathfrak{s}}^{\prime}}(i, j) \cap \mathrm{Cyl}_{\mathfrak{e}_{\mathfrak{s}^{\prime}}^{\prime}}(k, l) \text { if }\{i, j\}=\{1,2\}, \\ \left(S_{3} \cdot S_{4}\right) \cap \mathrm{Cyl}_{{\mathfrak{e _ { \mathfrak { s } } ^ { \prime }}}^{\prime}}(i, j) \cap \mathrm{Cyl}_{\mathfrak{e}_{\mathfrak{s}^{\prime}}^{\prime}}(k, l) \text { if }\{i, k\}=\{1,2\},\end{cases}
$$

where

$$
\begin{array}{ll}
S_{1}=\operatorname{Cyl}_{\mathfrak{i}_{\mathfrak{s}}}(i, 3) \cap \operatorname{Cyl}_{\mathfrak{i}_{\mathfrak{s}}}(j, 3) \cap \operatorname{Cyl}_{\mathfrak{i}_{\mathfrak{s}}}(4,3), \quad S_{2}=\operatorname{Cyl}_{\mathfrak{i}_{\mathfrak{s}}}(2, k) \cap \operatorname{Cyl}_{\mathfrak{i}_{\mathfrak{s}}}(2, l) \cap \operatorname{Cyl}_{\mathfrak{i}_{\mathfrak{s}}}(2,1), \\
S_{3}=\operatorname{Cyl}_{\mathfrak{i}_{\mathfrak{s}}}(i, 3) \cap \operatorname{Cyl}_{\mathfrak{i}_{\mathfrak{s}}}(4, k) \cap \operatorname{Cyl}_{\mathfrak{i}_{\mathfrak{s}}}(4,3), \quad S_{4}=\operatorname{Cyl}_{\mathfrak{i}_{\mathfrak{s}}}(j, 1) \cap \operatorname{Cyl}_{\mathfrak{i}_{\mathfrak{s}}}(2, l) \cap \mathrm{Cyl}_{i_{\mathfrak{s}}}(2,1) .
\end{array}
$$

Now the statement about double collinearity relations follows from the fact that any cylindricity relation is a relation of the scheme $\hat{\mathcal{C}}$.

Thus, we have $\mathcal{C}^{\prime} \geq \hat{\mathcal{C}}$. Recalling (29), we see that statement 1) of the theorem is proved. To prove statement 2), let $R \in \mathcal{R}(\mathcal{C})$. Then, obviously, the configuration $\Gamma=\operatorname{Der}\left(x_{u, v}, \mathcal{P}\right)$ does not depend on the choice of $(u, v) \in R$, where $x_{u, v}=(u, u, v, v)$, and

$$
R^{\delta}=\left\{x_{u, v}:(u, v) \in R\right\}=R(\Gamma, \mathcal{P})
$$

(see (11) ). However, statement 1$)$ implies that $R(\Gamma, \mathcal{P})$ is a basis relation of the scheme $\widehat{\mathcal{C}}$. Statement 2) is proved.

\section{§10. Proof of Theorem 1.4}

We deduce Theorem 1.4 from the following result to be used also in $\$ 11$.

Theorem 10.1. Let $\mathcal{C}$ and $\mathcal{D}$ (respectively, $\mathcal{C}^{\prime}$ and $\mathcal{D}^{\prime}$ ) be the nonhomogeneous and homogeneous schemes of a projective plane $\mathcal{P}\left(\right.$ respectively, $\left.\mathcal{P}^{\prime}\right)$, and let $\psi_{0}: \mathcal{C} \rightarrow \mathcal{C}^{\prime}$ and $\psi: \mathcal{D} \rightarrow \mathcal{D}^{\prime}$ be the canonical similarities. Suppose that a nontrivial similarity $\varphi$ of $\mathcal{C}$ is an m-similarity for some positive integer $m$. Then

1) $\widehat{\mathcal{D}}=\widehat{\mathcal{C}}^{\hat{\Phi}}$, where $\widehat{\mathcal{C}}=\widehat{\mathcal{C}}^{(m)}, \widehat{\mathcal{D}}=\widehat{\mathcal{D}}^{(m)}$, and $\widehat{\Phi}=\left\{1_{\hat{\mathcal{C}}}, \hat{\varphi}\right\}$ with $\hat{\varphi}=\hat{\varphi}^{(m)}$,

2) $\psi_{0} \in \operatorname{Sim}_{m}\left(\mathcal{C}, \mathcal{C}^{\prime}\right)$ if and only if $\psi \in \operatorname{Sim}_{m}\left(\mathcal{D}, \mathcal{D}^{\prime}\right)$.

Proof. It is easily seen that $\widehat{\mathcal{C}} \geq \widehat{\mathcal{D}}$. Since, obviously, the group $\widehat{\Phi}$ leaves fixed the set $\Delta_{m}$ and each basis relation of $\mathcal{D}^{m}$, it follows that

$$
\widehat{\mathcal{D}} \leq \widehat{\mathcal{C}}^{\widehat{\Phi}} \leq \widehat{\mathcal{C}} .
$$

Furthermore, the scheme $\mathcal{D}$ contains the equivalence relation $V_{\mathfrak{p}}^{2} \cup V_{\mathfrak{l}}^{2}$. In view of Theorem 3.2 if $R \in \mathcal{R}(\widehat{\mathcal{D}})$ and $i, j \in\{1, \ldots, m\}$, then either $t_{1}\left(x_{i}\right)=t_{1}\left(x_{j}\right)$ for all $x \in R$, or 
$t_{1}\left(x_{i}\right) \neq t_{1}\left(x_{j}\right)$ for all $x \in R$. So, for any $\mathfrak{s} \in \mathfrak{T}_{1}^{m}$ and any $\delta \in\{0,1\}^{m}$ the scheme $\hat{\mathcal{D}}$ contains the set $X_{\mathfrak{s}} \cup X_{\mathfrak{s}^{\prime}}$, where

$$
X_{\mathfrak{s}}=\left\{x \in V^{m}: t_{1}\left(x_{i}\right)=\mathfrak{s}_{i}, i=1, \ldots, m\right\}
$$

and $\mathfrak{s}^{\prime}$ is obtained from $\mathfrak{s}$ by replacing $\mathfrak{s}_{i}$ with its dual $\mathfrak{s}_{i}^{\prime}$ for all $i$, and also $\widehat{\mathcal{D}}$ contains the relation

$$
R_{\delta}=\left\{(x, y): \delta_{x_{i}, y_{i}}=\delta_{i}, i=1, \ldots, m\right\}=\bigcup_{\mathfrak{s} \in \mathfrak{T}_{1}^{m}} X_{\mathfrak{s}} \times X_{\overline{\mathfrak{s}}},
$$

where $\overline{\mathfrak{s}} \in \mathfrak{T}_{1}^{m}$ is such that $\delta_{\overline{\mathfrak{s}}_{i}, \mathfrak{s}_{i}}=\delta_{i}$ for all $i$ (the symbol $\delta$ in the expressions $\delta_{x_{i}, y_{i}}$ and $\delta_{\overline{\mathfrak{s}}_{i}, \mathfrak{s}_{i}}$ stands for the Kronecker delta). Since the relation $E=R_{\delta_{0}}$ with $\delta_{0}=(0, \ldots, 0)$ is an equivalence relation on $V^{m}$ with classes $X_{\mathfrak{s}}$, and the degree of the relation $\left(R_{\delta}\right)_{V^{m} / E}$ equals 1 for all $\delta$, this implies that the scheme $\widehat{\mathcal{D}}_{V^{m} / E}$ is semiregular. Applying Theorem 2.1 with $\mathcal{C}=\hat{\mathcal{D}}$, we conclude that

$$
\widehat{\mathcal{D}}=\left(\widehat{\mathcal{D}}_{E}\right)^{\Psi},
$$

where $\Psi$ is as in the theorem. In our case, the homogeneity sets of the scheme $\widehat{\mathcal{D}}_{V^{m} / E}$ are of the form $\left\{X_{\mathfrak{s}}, X_{\mathfrak{s}^{\prime}}\right\}$, where $\mathfrak{s} \in \mathfrak{T}_{1}^{m}$. So $|\Psi|=2$.

Clearly, $\hat{\mathcal{D}}$ contains any relation of the form $\bigotimes_{i=1}^{m}\left(R_{i} \cup R_{i}^{\varphi}\right)$, where $R_{i} \in \mathcal{R}(\mathcal{C})$ for all $i$. Moreover, by (31) we have

$$
\bigotimes_{i=1}^{m} R_{i}=\left(\bigotimes_{i=1}^{m}\left(R_{i} \cup R_{i}^{\varphi}\right)\right) \cap\left(X_{\mathfrak{s}} \times X_{\overline{\mathfrak{s}}}\right),
$$

where $\mathfrak{s}$ and $\overline{\mathfrak{s}}$ are determined uniquely from the condition $R_{i} \subset V_{\mathfrak{s}_{i}} \times V_{\overline{\mathfrak{s}}_{i}}$. So, $\widehat{\mathcal{D}}_{E} \geq \mathcal{C}^{m}$. Recalling that $\hat{\mathcal{D}}_{E}$ also contains $\Delta_{m}$, we conclude that

$$
\widehat{\mathcal{D}}_{E}=\hat{\mathcal{C}} \text {. }
$$

Thus, by (30) and (33), we have $\widehat{\mathcal{D}}=\hat{\mathcal{C}}^{\Psi} \leq \hat{\mathcal{C}}^{\hat{\Phi}}$. Since the groups $\Psi$ and $\widehat{\Phi}$ are of order 2 and act on $\mathcal{R}(\widehat{\mathcal{C}})$ without fixed points, this implies that $\Psi=\widehat{\Phi}$, whence statement 1 ) follows.

To prove statement 2), let $\psi_{0} \in \operatorname{Sim}_{m}\left(\mathcal{C}, \mathcal{C}^{\prime}\right)$. Set $\widehat{\psi}_{0}={\widehat{\psi_{0}}}^{(m)}$. Then, given $R, S \in$ $\mathcal{R}(\mathcal{C})$, we have

$$
\left(\bigotimes_{i=1}^{m}\left(R_{i} \cup R_{i}^{\varphi}\right)\right)^{\widehat{\psi_{0}}}=\bigotimes_{i=1}^{m}\left(R_{i}^{\psi_{0}} \cup R_{i}^{\varphi \psi_{0}}\right)=\bigotimes_{i=1}^{m}\left(R_{i}^{\psi_{0}} \cup R_{i}^{\psi_{0} \varphi^{\prime}}\right),
$$

where $\varphi^{\prime}$ is the nontrivial similarity of $\mathcal{C}^{\prime}$ (we have used the obvious relation $\varphi \psi_{0}=\psi_{0} \varphi^{\prime}$ ). Since also $\left(\Delta_{m}\right)^{\widehat{\psi_{0}}}=\Delta_{m}^{\prime}$, the restriction of $\widehat{\psi_{0}}$ to $\mathcal{R}(\widehat{\mathcal{D}})$ is the the $m$-extension of $\psi$. Thus, $\psi \in \operatorname{Sim}_{m}\left(\mathcal{D}, \mathcal{D}^{\prime}\right)$. Conversely, let $\psi \in \operatorname{Sim}_{m}\left(\mathcal{D}, \mathcal{D}^{\prime}\right)$. Then Theorem 3.2 shows that

$$
\left(X_{\mathfrak{s}} \cup X_{\mathfrak{s}^{\prime}}\right)^{\hat{\psi}}=X_{\mathfrak{s}}^{\prime} \cup X_{\mathfrak{s}^{\prime}}^{\prime}, \quad\left(R_{\delta}\right)^{\hat{\psi}}=R_{\delta}^{\prime}, \quad \mathfrak{s} \in \mathfrak{T}_{1}^{m}, \delta \in\{0,1\}^{m},
$$

where $\widehat{\psi}=\widehat{\psi}^{(m)}$ and $X_{\mathfrak{s}}^{\prime}$ and $R_{\delta}^{\prime}$ are defined by formulas (31) and (32) with $\mathcal{P}$ replaced by $\mathcal{P}^{\prime}$. We observe that the semiregular scheme $\mathcal{D}_{V^{m} / E}$ is generated by its homogeneity sets $\left\{X_{\mathfrak{s}}, X_{\mathfrak{s}^{\prime}}\right\}$ and the relations $\left(R_{\delta}\right)_{V^{m} / E}$ with full support. Therefore, by (34) and (36), the similarity $\widehat{\psi}_{V^{m} / E}$ is induced by a bijection $f: X_{\mathfrak{s}} \mapsto X_{\mathfrak{s}}^{\prime}$. So, by statement 4) of Theorem 2.1, the similarity $\widehat{\psi}$ can be extended to a similarity $\widehat{\psi}_{E}: \mathcal{D}_{E} \rightarrow \mathcal{D}_{E^{\prime}}^{\prime}$ such 
that $\left(\widehat{\psi}_{E}\right)_{V^{m} / E}$ is induced by $f$. Then from (34) it follows that

$$
\begin{aligned}
\left(\bigotimes_{i=1}^{m} R_{i}\right)^{\hat{\psi}_{E}} & =\left(\bigotimes_{i=1}^{m}\left(R_{i} \cup R_{i}^{\varphi}\right)\right)^{\hat{\psi}_{E}} \cap\left(X_{\mathfrak{s}} \times X_{\overline{\mathfrak{s}}} \hat{\psi}_{E}\right. \\
& =\bigotimes_{i=1}^{m}\left(R_{i} \cup R_{i}^{\varphi}\right)^{\psi} \cap\left(X_{\mathfrak{s}}^{f} \times X_{\mathfrak{s}}^{f}\right) \\
& =\bigotimes_{i=1}^{m}\left(R_{i} \cup R_{i}^{\varphi}\right)^{\psi_{0}} \cap\left(X_{\mathfrak{s}}^{\prime} \times X_{\mathfrak{s}}^{\prime}\right) \\
& =\bigotimes_{i=1}^{m}\left(R_{i}^{\psi_{0}} \cup R_{i}^{\psi_{0} \varphi^{\prime}}\right) \cap\left(X_{\mathfrak{s}}^{\prime} \times X_{\mathfrak{s}}^{\prime}\right) \\
& =\bigotimes_{i=1}^{m}\left(R_{i}^{\prime} \cup\left(R_{i}^{\prime}\right)^{\varphi^{\prime}}\right) \cap\left(X_{\mathfrak{s}}^{\prime} \times X_{\mathfrak{s}}^{\prime}\right) \\
& =\bigotimes_{i=1}^{m} R_{i}^{\prime} .
\end{aligned}
$$

Moreover, obviously $\left(\Delta_{m}\right)^{\hat{\psi}_{E}}=\Delta_{m}^{\prime}$, and $\mathcal{D}_{E}=\widehat{\mathcal{C}}, \mathcal{D}_{E^{\prime}}^{\prime}=\widehat{\mathcal{C}}^{\prime}$ (see (35)). Thus, $\hat{\psi}_{E}$ is the $m$-extension of $\psi_{0}$, whence $\psi_{0} \in \operatorname{Sim}_{m}\left(\mathcal{C}, \mathcal{C}^{\prime}\right)$.

Now statements 1) and 3) of Theorem 1.4 follow from Theorem 1.3 and Theorem 10.1 with $m=2$, whereas statement 2) is a consequence of statement 1) and the 2-closedness of the scheme $\mathcal{C}$ (statement 2) of Theorem 1.2).

\section{§11. Proof of Theorem 1.7}

For a projective plane $\mathcal{P}$ with element set $V$ and $\mathfrak{s} \in \mathfrak{T}_{1}$, we define a binary relation on $V^{3}$ as follows:

(37) $C_{\mathfrak{s}}=\left\{(x, y) \in T_{\mathfrak{s}} \times T_{\mathfrak{s}}: \exists v \in V: t_{1}(v)=\mathfrak{s}\right.$ and $v, x_{i}, y_{i}$ are collinear, $\left.i=1,2,3\right\}$,

where $T_{\mathfrak{s}} \subset V^{3}$ consists of all triples of noncollinear elements of type $\mathfrak{s}$ (see the footnote on page 85). Treating the elements of $T_{\mathfrak{p}}$ and $T_{\mathfrak{l}}$ as triangles in $\mathcal{P}$, we see that $C_{\mathfrak{p}}$ and $C_{\mathfrak{l}}$ consist of all pairs of triangles that are in perspective from some point and from some line, respectively. Thus, $\mathcal{P}$ is a Desarguesian plane 6 if and only if

$$
C_{\mathfrak{p}}=E \cdot C_{\mathfrak{l}} \cdot E^{T},
$$

where

$$
E=\left\{(x, y) \in T_{\mathfrak{p}} \times T_{\mathfrak{l}}: y_{1}=x_{2} x_{3}, y_{2}=x_{3} x_{1}, y_{3}=x_{1} x_{2}\right\} .
$$

We observe that the finite Desarguesian projective planes are exactly the Galois planes [14, §6]. On the other hand, it is easily seen that

$$
\begin{aligned}
C_{\mathfrak{s}} & =R \cdot R^{T}, \quad \Delta\left(T_{\mathfrak{s}}\right)=\Delta\left(V_{\mathfrak{s}}^{3}\right) \backslash\left(S_{\mathfrak{s}} \cdot S_{\mathfrak{s}}^{T}\right), \\
E & =\left(\bigcap_{1 \leq i \neq j \leq 3} \operatorname{Cyl}_{\mathfrak{i}_{\mathfrak{s}}}(i, j+3)\right) \cap\left(T_{\mathfrak{p}} \times T_{\mathfrak{l}}\right),
\end{aligned}
$$

\footnotetext{
${ }^{6}$ A Desarguesian plane is a plane for which the Desargues theorem holds true; for the details, see [14] p. 109].
} 
where

$$
\begin{aligned}
R & =\left(\bigcap_{1 \leq i \leq 3} \operatorname{Cyl}_{\mathfrak{i}_{\mathfrak{s}}}(i, i+3)\right) \cap\left(T_{\mathfrak{s}} \times\left(V_{\mathfrak{s}}^{3} \backslash T_{\mathfrak{s}}\right)\right), \\
\Delta\left(V_{\mathfrak{s}}^{3}\right) & =\bigcap_{1 \leq i \leq 3} \operatorname{Cyl}_{\mathfrak{e}_{\mathfrak{s}}}(i, i+3), \\
S_{\mathfrak{s}} & =\operatorname{Cyl}_{\mathfrak{i}_{\mathfrak{s}}}(1,4) \cap \mathrm{Cyl}_{\mathfrak{i}_{\mathfrak{s}}}(2,4) \cap \mathrm{Cyl}_{\mathfrak{e}_{\mathfrak{s}}}(3,4) .
\end{aligned}
$$

From now on we assume that $\mathcal{P}$ is a Galois plane. Then a nontrivial similarity of the nonhomogeneous scheme $\mathcal{C}$ of $\mathcal{P}$ is induced by any polarity of this plane. So, it is an $m$-similarity for all $m \geq 1$. Now, let $\varphi: \mathcal{C} \rightarrow \mathcal{C}^{\prime}$ be a 3 -similarity. Then without loss of generality we may assume that $\mathcal{C}^{\prime}$ is the scheme of a projective plane $\mathcal{P}^{\prime}$ and that the similarity $\varphi$ is canonical.

Theorem 3.2 shows that $C_{\mathfrak{s}}$ and $E$ (respectively, $C_{\mathfrak{s}}^{\prime}$ and $E^{\prime}$ ) are relations of the scheme $\widehat{\mathcal{C}}^{(3)}$ (respectively, $\widehat{\mathcal{C}}^{(3)}$ ), where $C_{\mathfrak{s}}^{\prime}$ and $E^{\prime}$ are the binary relations defined for $\mathcal{P}^{\prime}$ by (37) and (39), respectively. Moreover, the same theorem implies that, given $\mathfrak{s} \in\{\mathfrak{p}, \mathfrak{l}\}$, we have

$$
\left(C_{\mathfrak{s}}\right)^{\hat{\varphi}}=C_{\mathfrak{s}}^{\prime}, \quad E^{\hat{\varphi}}=E^{\prime},
$$

where $\hat{\varphi}=\hat{\varphi}^{(3)}$. On the other hand, since $\mathcal{P}$ is a Galois plane, (38) is fulfilled in $\mathcal{C}$, and hence in $\mathcal{C}^{\prime}$. Thus $\mathcal{P}^{\prime}$ is also a Galois plane. Since $\mathcal{P}$ and $\mathcal{P}^{\prime}$ are of the same order, they are isomorphic. Since the similarity $\varphi$ is canonical, it is induced by any isomorphism of these planes. Thus, $\operatorname{Sim}_{3}\left(\mathcal{C}, \mathcal{C}^{\prime}\right)=\operatorname{Sim}_{\infty}\left(\mathcal{C}, \mathcal{C}^{\prime}\right)$, whence $s(\mathcal{C}) \leq 3$.

Let $\mathcal{D}$ be the homogeneous scheme of the plane $\mathcal{P}$ and $\psi: \mathcal{D} \rightarrow \mathcal{D}^{\prime}$ a 3-similarity. Without loss of generality we assume that $\mathcal{D}^{\prime}$ is a homogeneous scheme of a projective plane $\mathcal{P}^{\prime}$ and that the similarity $\psi$ is canonical. Since the nontrivial similarity of $\mathcal{C}$ is a 3 similarity (see above), Theorem 10.1 with $m=3$ implies that so is the canonical similarity $\psi_{0}: \mathcal{C} \rightarrow \mathcal{C}^{\prime}$, where $\mathcal{C}$ and $\mathcal{C}^{\prime}$ are nonhomogeneous schemes of $\mathcal{P}$ and $\mathcal{P}^{\prime}$, respectively. But by the first part of the same theorem, $\psi_{0}$ is induced by some isomorphism. Clearly, it also induces $\psi$.

\section{APPENDIX}

In the following table we enumerate all $\Xi$-configurations $\Gamma$ belonging to the set $\operatorname{Der}(\mathcal{P})$, where $\mathcal{P}$ is a projective plane of order $q$. Any such configuration satisfies the following conditions:

(a) any two vertices of $\Gamma$ belonging to the same class are equal (the type of the corresponding edge is $\mathfrak{e}$ );

(b) any two vertices of $\Gamma$ belonging to incident classes are incident;

(c) there are no quadrangles in $\Gamma$ (see $\$ \$$ and 5 ).

We represent a configuration as a bipartite graph, the parts of which consist of classes of the same type, and the edges correspond to incident classes (3rd column); the restriction of $\Gamma$ to $\Xi$ is represented in the 2nd column, whereas its informal description is in the 4th column. The label of a class is the cardinality of it; we omit the label if the class is a singleton. In the 5 th column of the table we write the number of $\Xi$-configurations represented by the graph in the corresponding row; the number in the 6th column shows that the corresponding row applies to a projective plane of order greater than or equal to that number. 


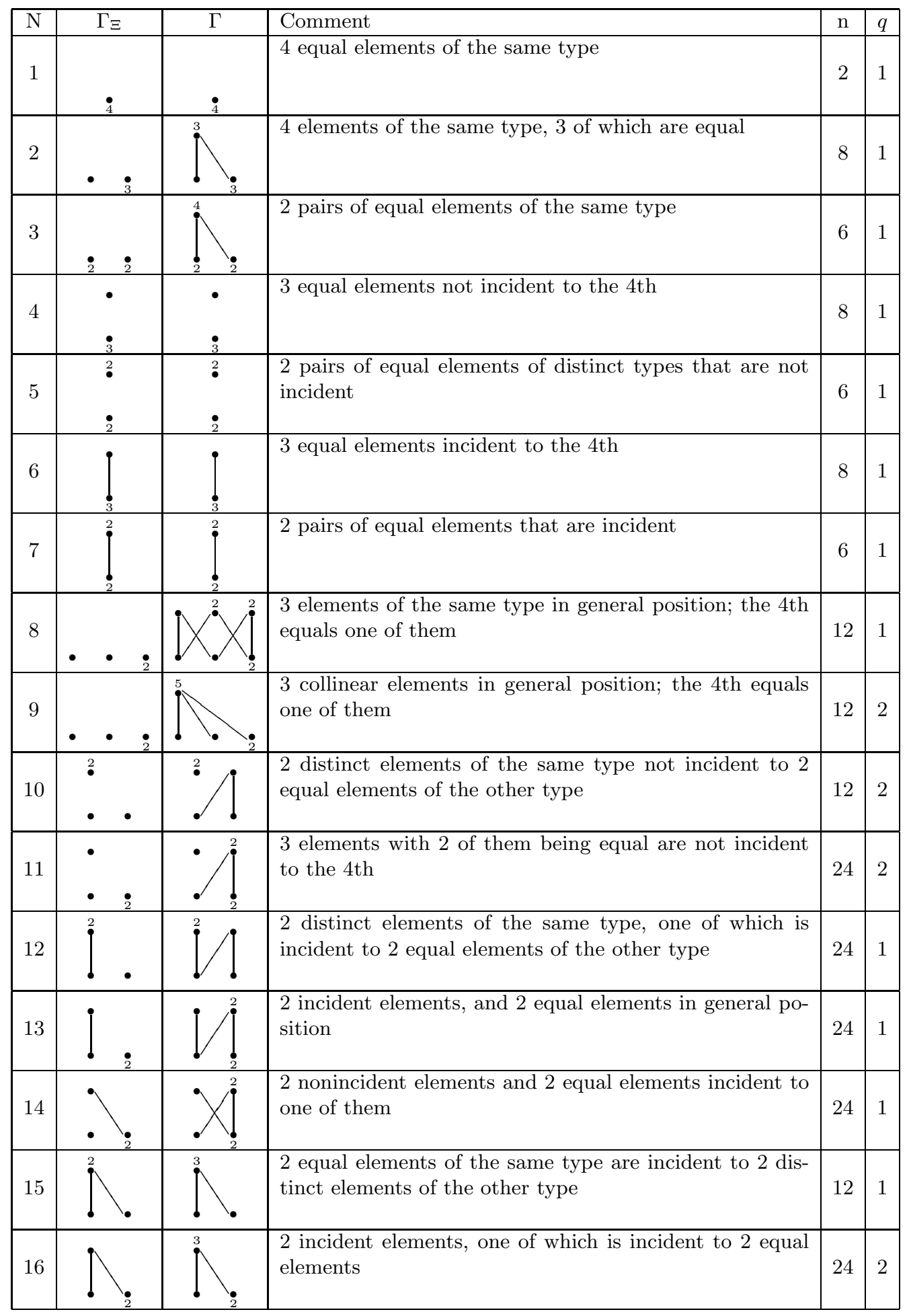




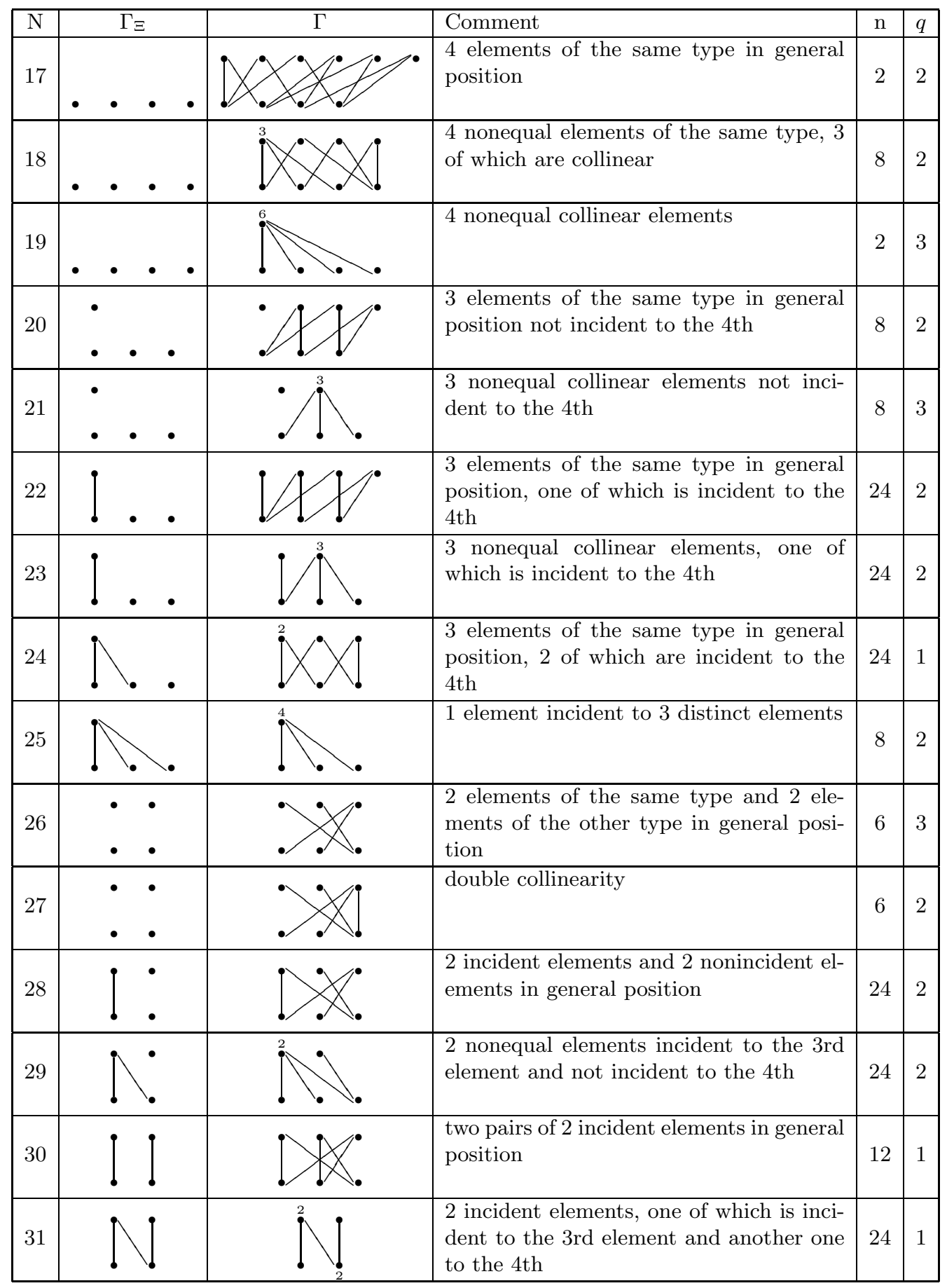




\section{REFERENCES}

[1] S. A. Evdokimov, Schurity and separability of associative schemes, Doctor's Thesis., S.-Peterburg. Gos. Univ., St. Petersburg, 2004. (Russian)

[2] S. A. Evdokimov and I. N. Ponomarenko, Characterization of cyclotomic schemes and normal Schur rings over a cyclic group, Algebra i Analiz 14 (2002), no. 2, 11-55; English transl., St. Petersburg Math. J. 14 (2003), no. 2, 189-221. MR1925880(2003h:20005)

[3] _ Rings of finite projective planes and their isomorphisms, Zap. Nauchn. Sem. S.-Peterburg. Otdel. Mat. Inst. Steklov. (POMI) 289 (2002), 207-213; English transl., J. Math. Sci. (N. Y.) 124 (2004), no. 1, 4792-4795. MR1949741 (2003g:51005)

[4] A. E. Brouwer, A. M. Cohen, and A. Neumaier, Distance-regular graphs, Ergeb. Math. Grenzgeb. (3), Bd. 18, Springer-Verlag, Berlin, 1989. MR1002568 (90e:05001)

[5] S. Evdokimov and I. Ponomarenko, On highly closed cellular algebras and highly closed isomorphisms, Electron. J. Combin. 6 (1999), Res. Paper 18, 31 pp. MR.1674742 (2000e:05160)

[6] _ Separability number and Schurity number of coherent configurations, Electron. J. Combin. 7 (2000), Res. Paper 31, 33 pp. MR1763969 (2001g:05108)

[7] S. Evdokimov, M. Karpinski, and I. Ponomarenko, On a new high-dimensional Weisfeiler-Lehman algorithm, J. Algebraic Combin. 10 (1999), no. 1, 29-45. MR1701282 (2001i:05110)

[8] I. A. Faradžev, Association schemes on the set of antiflags of a projective plane, Discrete Math. 127 (1994), 171-179. MR.1273600 (95e:05124)

[9] I. A. Faradžev, M. H. Klin, and M. E. Muzichuk, Cellular rings and groups of automorphisms of graphs, Investigations in Algebraic Theory of Combinatorial Objects, Math. Appl. (Soviet Ser.), vol. 84, Kluwer Acad. Publ., Dordrecht, 1994, pp. 1-152. MR.1273366 (95a:05049)

[10] D. G. Glynn, Rings of geometries. I, J. Combin. Theory Ser. A 44 (1987), no. 1, 34-48. MR0871387 (88g:51011)

[11] D. G. Higman, Characterization of families of rank 3 permutation groups by the subdegree. I, II, Arch. Math. (Basel) 21 (1970), 151-156; 353-361. MR0268260 (42:3159) MR0274565 (43:328)

[12] , Partial geometries, generalized quadrangles and strongly regular graphs, Atti del Convegno di Geometria Combinatoria e sue Applicazioni (Univ. Perugia, Perugia, 1970), Ist. Mat., Univ. Perugia, Perugia, 1971, pp. 263-293. MR0366698(51:2945)

[13] _ Coherent algebras, Linear Algebra Appl. 93 (1987), 209-239. MR0898557 (89d:15001)

[14] D. R. Hughes and F. C. Piper, Projective planes, Grad. Texts in Math., vol. 6, Springer-Verlag, Berlin, 1973. MR0333959(48:12278)

[15] M. Klin, M. Muzychuk, C. Pech, A. Woldar, and P. H. Zieschang, Association schemes on 28 points as mergings of a half-homogeneous coherent configuration, European J. Combin. 28 (2007), 1994-2025. MR2344983 (2008f:05211)

St. Petersburg Branch, Steklov Mathematical Institute, Russian Academy of Sciences, Fontanka 27, 191023 St. Petersburg, Russia

E-mail address: evdokim@pdmi.ras.ru

St. Petersburg Branch, Steklov Mathematical Institute, Russian Academy of Sciences, Fontanka 27, 191023 St. Petersburg, Russia

E-mail address: inp@pdmi.ras.ru

Received 18/APR/2008

Translated by THE AUTHORS 